\title{
1 A Combined Human Gastruloid Model of Cardiogenesis and
}

\section{Neurogenesis}

3 Zachary T. Olmsted ${ }^{1}$ and Janet L. Paluh ${ }^{1 *}$

$4 \quad{ }^{1}$ State University of New York Polytechnic Institute, College of Nanoscale Science and

5 Engineering, Nanobioscience, Albany, NY 12203

6

$7 \quad$ *Corresponding author:

Dr. Janet L. Paluh State University of New York Polytechnic Institute College of Nanoscale Science and Engineering 4424 NanoFab East 257 Fuller Road, Albany, NY 12203

Phone: (518) 956-7047

Fax: (518) 437-8687

Email: paluhi@sunypoly.edu 


\section{SUMMARY}

16 Multi-lineage development from gastruloids is enabling unprecedented opportunities to model and

17 study human embryonic processes and is expected to accelerate ex vivo strategies in organ

18 development. Reproducing human cardiogenesis with neurogenesis in a multi-lineage context

19 remains challenging, requiring spatiotemporal input of paracrine and mechanical cues. Here we

20 extend elongating multi-lineage organized (EMLO) gastruloids to include cardiogenesis (EMLOC)

21 and describe interconnected neuro-cardiac lineages in a single gastruloid model. Contractile

22 EMLOCs recapitulate numerous interlinked developmental features including heart tube

23 formation and specialization, cardiomyocyte differentiation and remodeling phases, epicardium,

24 ventricular wall morphogenesis, chamber-like structures and formation of a putative outflow tract.

25 The EMLOC cardiac region, which originates anterior to gut tube primordium, is progressively

26 populated by neurons in a spatial pattern mirroring the known distribution of neurons in the

27 innervated human heart. This human EMLOC model represents the first multi-lineage

28 advancement for the study of coincident neurogenesis and cardiogenesis. 


\section{INTRODUCTION}

33 The complex nature of in vivo cardiogenesis underlies the difficulties in establishing in vitro

34 cardiac developmental models with human cells. The heart is the first organ to form in the

35 mammalian embryo, caudal to the embryonic brain and within the developing trunk. It becomes

36 contractile as a tube prior to complex morphogenesis into septated chambers and co-

37 developmental population by neurons for innervation (Harvey, 2002; Hasan, 2013). In order to

38 accommodate both contractility and structural rearrangement, the developing heart undergoes

39 alternating phases of cardiac differentiation and morphogenesis (Ivanovitch et al., 2017). Calcium

40 handling properties become refined during cardiac differentiation (Tyser et al., 2016). The cardiac

41 crescent is the first bilateral structure to form and precedes epithelialization and formation of the

42 transversal heart tube. At this stage, the heart tube remains open at the dorsal aspect, bound by

43 dorsal mesocardium, and then seals during formation of the closed linear heart tube and outflow

44 tracts. Intrinsic cell-driven forces within the tube and extrinsic physical constraints are known to

45 mediate the establishment of left-right asymmetries required for heart function (Desgrange et al.,

46 2018). Such complexity in cardiogenesis lays the framework for lifelong functioning of the adult

47 heart, but also underlies the propensity for congenital heart disease in humans where

48 developmental errors induce cardiac malformations (van der Linde et al., 2011; Desgrange et al.,

49 2018). The ability to generate in vitro models of heart development that mimic essential aspects

50 of multi-lineage input to cardiogenesis will benefit biomedical treatments of heart disease and

51 progress towards ex vivo organogenesis.

Organoid technology is revolutionizing the study of human development and disease,

53 recapitulating key aspects of spatiotemporal tissue morphogenesis (Clevers, 2016; Olmsted and

54 Paluh, 2021c). Most current organoid technologies are directed towards single tissue endpoints

55 that lack the cellular contextual diversity present in normal organogenesis through inductive and

56 mechanical interactions. As such, the ability to generate organotypic human cardiac organoids

57 that form according to the in situ developmental signaling blueprint and integrate with the

58 developing nervous system has not been achieved. The existing human cardiac organoid models

59 derive primarily from pre-differentiated cardiomyocytes and their spheroid aggregates that form

60 irrespective of developmental timelines (Nguyen et al., 2014; Giacomelli et al., 2017; Polonchuk

61 et al., 2017; Andersen et al., 2018), or models that rely on integrated bioengineering efforts to

62 constrain morphogenetic patterning (Ma et al., 2015; Lind et al., 2017; Macqueen et al., 2018;

63 Hookway et al., 2019). These models lack identified critical inductive tissues indispensable to

64 natural heart development such as the foregut, described as a central organizer of cardiogenesis 
in multiple species and acting through both inductive and structural interactions between endoderm and splanchnic mesoderm (Nascone and Mercola, 1995; Schultheiss et al., 1995; Varner and Taber, 2012; Anderson et al., 2016; Kidokoro et al., 2018; Han et al., 2020).

Two recent studies with human iPSCs succeeded in the co-production of cardiac and gastrointestinal tissue in single organoids without organized chambers (Silva et al., 2021; Drakhlis et al., 2021). As well, Hofbauer et al. (2021) succeeded in generating self-organized, isolated cardioids exhibiting chamber-like structures from human pluripotent stem cells that were used to model cardiac injury (Hofbauer et al., 2021). Although important advances to the cardiogenesis field, neural cells were not co-generated in these systems and were absent. One murine study generated chambered cardiac organoids from mESCs by embedding in exogenous extracellular matrix (ECM) with supplied FGF4 (Lee et al., 2020). More recently, Rossi et al. (2021) used mESC-derived gastruloids to recapitulate aspects of early cardiogenesis including first and second heart field contributions without extracellular matrix (ECM) embedding. Gastruloid research has been broadly applicable for conducting multi-lineage interaction studies in the trunk (van den Brink et al., 2014; van den Brink et al., 2020; Veenvliet et al., 2020; Olmsted and Paluh, 2021a). However, no study with human cells has succeeded in generating a de novo model to recapitulate cardiogenesis in an embryo-like, multi-lineage context and, in particular, with neuronal cooperative development that is a vital functional component. Here we achieve this goal and describe human gastruloids that capture numerous key developmental aspects of human cardiogenesis and neurogenesis along with endoderm-derived primitive gut tube and other lineages.

We recently described a unique human trunk model system referred to as elongating multilineage organized (EMLO) gastruloids (Olmsted and Paluh, 2021a, 2021b). Neural crest lineage in EMLOs reveals insights into enteric development and the formation of the enteric nervous system. The enteric multi-lineage niche in EMLOs achieved only limited embryonic cardiogenesis that included generation of cardiomyocytes anterior to the gut tube. We therefore hypothesized that EMLOs could be coaxed developmentally, if provided the necessary cues, towards more

92 extended cardiac differentiation with reproducible morphogenesis. We reoptimized our EMLO 93 formation protocol to include angiocrine and pro-cardiogenic factors previously detailed (Rossi et 94 al., 2021). We now demonstrate a gastruloid strategy for neuro-cardiac co-developed tissues that 95 recapitulate aspects of early human heart morphogenesis with neuronal integration. We track 96 multiple events in cardiomyocyte differentiation from splanchnic mesoderm and observe 97 spontaneous contractility, chamber precursor formation, early constrictions and septations, 
epicardium, and putative structures resembling the outflow tracts. Critically, EMLOCs not only retain the interacting neural compartment but achieve neurogenesis to generate an organized codeveloped neuro-cardiac gastruloid. This work establishes EMLOCs as an advanced model for human cardiogenesis and the integration with endoderm and neurons towards the goal of organ innervation.

\section{RESULTS}

Angiocrine and pro-cardiogenic factors redirect multi-lineage EMLO gastruloids for optimized human developmental cardiogenesis (EMLOCs). We previously generated EMLO gastruloids with co-developing central and peripheral neurons and trunk mesendoderm including components of the enteric nervous system (Olmsted and Paluh, 2021a, 2021b). To test the ability of EMLOs to model human developmental cardiogenesis (EMLOCs), we modified exposure to growth factors during early formation and polarization stages in a revised protocol (Figure 1A, Figure S1). EMLOCs were generated with the hiPSC line H3.1.1 that we previously validated for differentiation into contractile cardiomyocytes (Tomov et al., 2016) and that forms EMLOs by the original protocol described (Olmsted and Paluh, 2021a, 2021b). EMLOCs were handled identically to EMLOs during a 2D induction phase up to $48 \mathrm{~h}$ post-aggregation in shaking cultures. At $48 \mathrm{~h}$, the N2B27 supplemented with $10 \mathrm{ng} / \mathrm{ml} \mathrm{FGF2,} 2 \mathrm{ng} / \mathrm{ml} \mathrm{HGF}$, and $2 \mathrm{ng} / \mathrm{ml}$ IGF-1 in EMLOs was instead supplemented with $30 \mathrm{ng} / \mathrm{ml}$ FGF2, $5 \mathrm{ng} / \mathrm{ml} \mathrm{VEGF}$, and $0.5 \mathrm{mM}$ ascorbic acid (AA) (Figure S1A-C) as was done for the mESC cardiac gastruloid model (Rossi et al., 2021). The new cardiogenic factors were added in the absence of the initial factors used for induction. The EMLOC gastruloids were maintained in this pro-cardiogenic medium to day 7 . In the first $48 \mathrm{~h}$ prior to the medium change, early germ layer biomarkers were expressed in appropriately sized gastruloids as expected for the EMLO protocol. That is, uniform expression of SOX2 $24 \mathrm{~h}$ post-

122 aggregation, with little to no expression of GATA6 and heterogenous expression of FOXA2 123 (Figure S1D). Mitotically active cells in the gastruloid were visible (Figure S1E). After the medium 124 change, the EMLOC gastruloids that were continually maintained in shaking culture began to 125 elongate by day 5 . By day 7 , elongated gastruloids formed thin-walled, dilated chamber-like 126 structures with spontaneous contractility (Figure 1; Movies S1 and S2). Partitioning of 127 cardiogenic chamber precursors was visible by phase contrast microscopy (Figure S1F), and 128 validated by expression of the early cardiogenic transcription factor GATA6 along with cardiac 129 Troponin T (cTnT) (Figure S1G). Multiple distinct cavities resembling chambers were also 130 identified by immunofluorescence in single EMLOCs with early evidence of septation (Figures 
131 1B and 1C). These findings support the ability to direct cardiogenic tissue precursors within

132 gastruloids by early manipulation of growth conditions and signaling factors, demonstrated by the

133 genesis of self-organizing cardiogenic compartments. We next performed single cell RNA

134 sequencing (scRNAseq) to further delineate the cell and tissue precursor types generated by this

135 protocol.

EMLOC gastruloids generate diverse embryonic cell types of the human trunk revealed by scRNAseq analysis. Single cell sequencing of H3.1.1 derived EMLOCs was performed at two time points that are day 7 and day 16 after initial aggregation in shaking culture. The integrated dataset of both time points was analyzed (2,859 cells) (Figures 1D-1F) along with each time point individually (day 7: 1,004 cells; day 16: 1,855 cells) (Figure 2, Figure S2). The integrated dataset was generated in Seurat and visualized using UMAP and PHATE methods. Ten clusters were produced and were annotated using differentially expressed genes (DEGs) and cell or tissue type characteristic biomarkers from the literature (Figures 1D and 1E). The top five DEGs for each cluster of the integrated dataset is provided (Figure 1F) in addition to a comprehensive list of DEGs and genes used for cluster annotation (Table S1; Supplementary data file). Clusters were annotated as trunk neuroectoderm/spinal cord progenitors (cluster 1), trunk neurons (cluster 8), peripheral glia/Schwann cells (cluster 10), mitotic cells (cluster 5), mixed cell types (cluster 0), cardiomyocytes (CM; cluster 3), epicardial cells and cardiac fibroblasts (EP, CF; clusters 2 and 7 ), intermediate mesoderm and metanephric mesenchyme (IM; cluster 4), genitourinary/renal epithelium (GU; cluster 6), and vascular endothelium (VE; cluster 9). By PHATE analysis of day 16 cells, we visualized distinct cardiac, epithelial and neural lineages (Figure 2A). We overlaid important signaling pathways including BMP, SHH, and WNT signaling that were delineated along distinct lineages (Figure 2B). BMP4 was highly expressed in the cardiac region while BMP7 bifurcated along neural and trunk epithelial lineages. $\mathrm{SHH}$ was upregulated in a region within cluster 0 of anterior foregut (AFG) phenotype (FOXA2, NKX2-1, SHH, EPCAM) that is a known developmental organizer of cardiogenesis (Anderson et al., 2016). WNT2B expression localized to the cardiac region while WNT1 and WNT3A localized to the spinal cord region with known 159 involvement in neural tube morphogenesis and neural crest patterning. WNT1 is shown. Cadherin 160 and HOX genes were also delineated along respective lineages, consistent with developmental 161 cadherin and HOX codes in vivo (Figure 2C). $C D H 11$ was upregulated in the cardiac region while $162 \mathrm{CDH6}$ was upregulated in neuroectoderm and mesenchyme and $\mathrm{CDH1}$ was upregulated in 163 epithelium. Distinct expression of HOXC6 and HOXC9 in neural clusters was indicative of caudal 164 neuraxis and trunk spinal cord. HOXD8 and HOXD9 were specific to epithelium with renal mRNA 
expression phenotype (cluster 6) while HOXA4 was predominantly expressed in the cardiac region. This scRNAseq analysis reflects a diversity of cell and tissue precursor types generated within EMLOCs with signaling networks, adhesion proteins, and transcription factors mirroring in vivo development. We elaborate on specific features of the annotated clusters throughout the manuscript where indicated, with emphasis on neural, cardiac, and foregut endodermal lineages.

\section{Multiple derivatives of splanchnic mesoderm in EMLOCs identified by scRNAseq.}

172 Cardiogenic mesoderm gives rise not only to working cardiomyocytes but also contributes to 173 epicardium, endocardium, connective tissue, outflow tract, valves and the conduction apparatus.

174 Cluster annotation and analysis of scRNAseq data in day 7 and day 16 EMLOCs identified diverse 175 cell types involved in cardiogenesis arising from splanchnic mesoderm (Figures 2D-2E, Figure 176 S3) including cardiomyocytes that form myocardium, cells of the proepicardium and epicardium, and cardiac fibroblasts, in addition to endocardium and vascular endothelium. In mouse, cardiac precursors arise within the splanchnic mesoderm and differentiate into cardiomyocytes by assembling contractile machinery (Tyser et al., 2016). EMLO gastruloids were previously shown to contain splanchnic mesoderm permissive to cardiomyocyte differentiation (Olmsted and Paluh, 2021a). Similarly, EMLOCs retain characteristic expression of the splanchnic mesoderm biomarkers FOXF1, PDGFRA, TWIST1, and PRRX2 (Figure S3A). GATA4 and GATA6 that are indispensable for cardiogenesis were expressed in a similar distribution (Figure S3B). Sarcomeric proteins associated with cardiomyocytes such as TNNT2 (cardiac troponin), TNNT1 (slow type troponin) and MYL7 were most localized to cluster 3 at day 16 along with first heart field (FHF) and second heart field (SHF) biomarkers TBX5 and HAND2 (Figure 2D). Additional FHF genes (NKX2-5, HAND1) and SHF genes (MEF2C, ISL1, TBX18) were similarly distributed. At the day 16 time point, cardiomyocytes were primarily of ventricular cell phenotype. DEGs involved in regulating ventricle growth, morphogenesis, and contractility were identified (Figure S3B). Cluster 3 contained additional upregulated genes involved in fetal heart development such as dimeric KRT8/KRT18, APOE, PLAC9 and S100A10. These genes were also upregulated in cluster 2 that were annotated as epicardial (EP) cells based on expression profile (WT1, TCF21, TPJ1, LHX2, LHX9, TBX18, PLAC9) (Figure 2E). Cluster 7 had a similar expression profile to 194 cluster 2, but with several distinguishing features such as reduced proliferation assessed by 195 MKI67, upregulation of IGFBP5 associated with cardiac fibroblast activation and BTS2 that 196 interacts with IFI16, a biomarker of mature cardiac fibroblasts. A previous analysis of human fetal 197 cardiogenesis revealed subclusters of actively proliferating, early fibroblasts and those enriched 198 for ECM organization with less proliferation (Cui et al., 2019). A similar observation was made 
here between cluster 2 (high proliferation by MKI67) and cluster 7 (low proliferation by MKI67). The scRNAseq clusters representing cardiogenesis expressed a characteristic milieu of cardiomyocyte and fibroblast-derived ECM genes (Figure S4). Cluster 0 contained upregulated genes indicating a mixture of cell types from cardiogenic mesoderm including contractile cells (TNNT2, MYL7), outflow tract cells (PDE5A, ISL1, FN1, MEGF6, MSX2, SEMA3C, EMILIN1, CNN1, TAGLN), and cells involved in atrioventricular conduction and organization (GJA1, CACNA1H, TBX3, CXCL12, DSP). Anterior foregut progenitor cells were also identified (HHEX, $S H H, F O X A 2)$. Cells expressing cardiac neural crest biomarkers (ETS1, EDNRA, TGIF1, HOXA3) were dispersed throughout the four clusters (Figure S3C). Cardiac neural crest cells in vivo play critical roles in cardiogenesis and organization, including valve and outflow tract contributions. Regulatory subnetworks within EMLOCs therefore generate a range of the cell types involved in cardiogenesis along with an appropriate ECM milieu.

\section{Human EMLOC multi-lineage gastruloids form chamber-like structures with spontaneous} contractility and calcium signaling. Intracellular changes in $\mathrm{Ca}^{2+}$ couple cardiomyocyte depolarization with contraction. To demonstrate that EMLOCs express calcium-regulated contractile proteins and achieve calcium-mediated contractility, we performed 3D fixed and live cell imaging analysis (Figure 3). The cardiogenic compartment in EMLOCs exhibited visible sarcomeres using cTnT immunofluorescence (Figure 3A) and spontaneous contractility (Figure 3B). To quantify spontaneous contractility, we compared beating phenotypes in EMLOC-directed gastruloids versus the original EMLO protocol that is not optimized for cardiogenesis (Olmsted and Paluh, 2020a) $\left(\mathrm{N}=3\right.$ repeat experiments; ${ }^{* *} \mathrm{p}=0.0005, \mathrm{t}=10.40 \mathrm{df}=4$ by unpaired twotailed t-test). We further performed live calcium imaging with Fluo-4 AM (Figures 3C-3E). In

222 Figure 3C, we demonstrate robust Fluo-4 AM activity in the contractile region of two gastruloids

223 and quantify F/Fo calcium transients (Figure 3D; Movies S3 and S4). The EMLOCs shown were

224 captured in the same field and are representative of the quantified population. Median calcium

225 transients per min (corresponding to beat frequency) was 10.5 ( $n=10$, $\max =19$, $\min =5$, median

$226=10.5, q 1=8, q 3=12.75$ ) (Figure $3 E$ ). Known genes involved in cardiac action potential 227 conduction and calcium handling are expressed, including $I R X 3$ and $I R X 5$ that play roles in rapid 228 ventricular conduction and cardiac repolarization, respectively, along with the ITPR2 calcium 229 channel and sodium-calcium exchanger SLC8A1/NAC1 that were all expressed with similar 230 distribution (Figure 3F). Together these data demonstrate that morphological cardiogenesis 231 chamber features, calcium signaling and spontaneous contractility can be directed in human 
232 EMLOCs by modifying the EMLO protocol with exposure to angiocrine and pro-cardiogenic 233 growth factors.

Recapitulating early morphogenesis events in human developmental cardiac EMLOC gastruloids. The first cardiogenic structure to form in the anterior aspect of the mammalian embryo is called the cardiac crescent, which fuses to form the transversal heart tube that seals dorsally to generate a closed tube with outflow tracts (Figure 4A). We identified cardiac crescentlike structures at high penetrance in EMLOC (73.0 +/- 7.2\%, mean +/- s.e.m.) versus EMLO generated gastruloids $\left(2.7+/-1.8 \% ; \mathrm{N}=3\right.$ repeat experiments; ${ }^{* * *} \mathrm{p}=0.0007, \mathrm{t}=9.474, \mathrm{df}=4$ by unpaired two-tailed t-test) (Figure 4B). Cardiac crescent regions at day 4 in EMLOC formation contained cTnT+ cardiomyocyte progenitors co-localized with GATA6, a transcription factor required for high fidelity cardiogenesis (Figures 4C and 4E). The cardiogenic region increased in size with time, extending laterally away from the main body of the gastruloid (Figure 4D). The same region developed a cell-free interior, resembling early heart tube formation (Figures 4A and 4F) along with cardiac chamber morphological precursors (Figure 4G).

EMLOC gastruloid cardiac morphogenesis occurs anterior to primitive gut tube endoderm. The developing anterior foregut derived from endoderm has been shown to be essential for cardiogenesis in multiple organisms through crosstalk with splanchnic mesoderm and by providing mechanical cues (Figure 5A) (Nascone and Mercola, 1995; Varner and Taber, 2012; Anderson et al., 2016; Kidokoro et al., 2018; Han et al., 2020). We previously demonstrated that the primitive gut tube can self-organize reproducibly in the EMLO gastruloid system (Olmsted and Paluh, 2020a). Therefore, we investigated whether this structure is present in the EMLOC gastruloids optimized for cardiogenesis. In mouse, this occurs anterior to the gut tube, including cuboidal epithelialization that is required for second heart field contributions to heart tube formation (Ivanovitch et al., 2017; Cortes et al., 2018). The splanchnic mesoderm is an established and reproducible feature of EMLO and EMLOC formation and also contributes to the gut tube (Figure S3A and S3B) (Olmsted and Paluh, 2021a). Using scRNAseq, a population of cells with anterior foregut identity (FOXA2, NKX2-1, SHH, EPCAM) was identified and clustered adjacent to the developing cardiac region (Figure 2A). Spatially organized FOXA2+ cells adjacent

262 to the cardiac region were also identified using immunofluorescence. By normalizing fluorescence 263 and gastruloid end-to-end length, an average profile for cTnT (cardiac) and FOXA2 (gut tube 264 endoderm) was generated over the anterior-posterior axis to depict relative positioning in day 4 265 EMLOCs (Figure 5B) (N = 5 EMLOCs). Representative cTnT/FOXA2 immunofluorescence Z- 
slices are provided for day 5 and day 6 EMLOCs, in addition to CDH1 (E-Cadherin) and GATA6/Type 1 Collagen (Figures 5C and 5D). These data prioritize visualization of gut tube positioning relative to the cardiogenic region, demonstrating the appropriate posterior embryological trunk spatial organization with respect to cardiogenesis. We performed immunofluorescence imaging with cTnT and the proliferation marker Ki67, as well as $\mathrm{CDH} 2$ that is essential for ventricular wall morphogenesis (Miao et al., 2019). The co-immunofluorescence

272 of the $\mathrm{CDH} 2$ biomarker with CTnT further revealed early organization of cells in the cardiac

273 crescent into epithelial-like cytoarchitectures that is a contributing factor in heart tube formation

274 (Figure 5E) (Cortes et al., 2018). This pattern was also observed by scRNAseq co-expression

275 patterns of $C D H 2$ with ventricular biomarkers (Figure S3B).

We further investigated whether EMLOCs recapitulate distinct phases of cardiomyocyte differentiation and morphogenesis that are ongoing developmentally (Figures S5). As done in vivo (Ivanovitch et al., 2017), cardiomyocyte shape was used to indirectly infer distinct phases of cardiomyocyte differentiation versus structural morphogenesis with cellular proliferation. We characterized cardiomyocyte cell shape as rounded (morphogenesis and proliferation; Figure S5A top) versus adhesive flat/mosaic (differentiation; Figure S5A bottom) and quantified the relative proportion of EMLOCs exhibiting one phenotype or the other in single day 7 fixed samples (Figure S5B) ( $N=4$ replicates; $34+/-18 \%$ round, range 16 to 59\%; $66+/-18 \%$ flat/mosaic, mean +/- s.e.m., range 41 to $84 \%$; n.s. $p=0.1776, t=1.754$, $d f=3$ by paired two-tailed $t$-test). Together, these data demonstrate that features of in vivo cardiogenesis can be modeled in EMLOCs within the appropriate multi-lineage gastruloid microenvironment. tube in vivo, specialization over the length of the tube establishes the future blueprints for the adult heart in terms of septated chambers and outflow tracts that transmit and receive blood (Figure 6A). Divisions of the embryonic heart are separated by minor constrictions in the tube.

293 We identified day 7 EMLOCs with cardiogenic compartments resembling this stage in heart tube 294 development (Figure 6B). After day 7, constricted tubes became dilated and had early divisions 295 between chamber precursors (Figures 6C and 6D), visualized by 3D reconstructions and multi296 dimensional analysis. The fluid-filled contractile cavities were completely surrounded by 297 continuous cTnT+ cardiomyocytes indicative of myocardium. In addition, the cavities had open 298 channels communicating with the posterior EMLOC compartment (Figures 6C and 7D, Figure 
7). Genes involved in left-right asymmetry specification during in vivo cardiogenesis were also upregulated in the day 16 scRNAseq data set (IRX3, HAND1, PITX2, RTTN).

In developing heart chambers in situ, the chamber walls are multi-layered, with myocardium composed of working contractile and conducting cardiomyocytes comprising the outermost layer, and endocardium lining comprising the innermost layer (Figure 7A). An epicardial membrane surrounds these layers. Myocardium and endocardium are initially separated by ECM-rich "cardiac jelly" (Kim et al., 2018) that is degraded with time, necessary for chamber morphogenesis. Using 3D image reconstruction and multi-dimensional analysis, we show that chamber wall organization was recapitulated in developing EMLOC chamber-like structures (Figure 7B). We identified gene biomarkers of cardiac jelly ECM and its spatiotemporal degradation VCAN, ADAMTS1, and ANGPT1 that were highly expressed in day 16 EMLOCs (Figure 7C) (Kim et al., 2018). Notably, an interior channel partially lined by cTnT+ cells was also identified with high penetrance, postulated here to be initiation of the putative outflow tract due to its appropriate positioning (Figure 7D) and that is lined by segmental CDH5 immunostaining extending out into the posterior compartment of the EMLOC (Figure 7E). By scRNAseq, we identified cells with combinations of biomarkers for smooth muscle (CNN1/TAGLN), outflow tract development (ISL1/PDE5A/CDH11), and well-differentiated vascular endothelium (cluster 9; $K D R / F L T 1 / E S A M / C D H 5)$ (Figure 7F). Nodal and valvar biomarkers were also present with a similar distribution (POSTN/TBX3/NPR3/NFATC4) (Figure 7G). These data, taken together, are consistent with cardiogenesis in EMLOCs proceeding in an appropriate spatiotemporal manner and detailed morphological and gene expression changes, according to aspects of in situ development.

EMLOCs capture neurogenesis within a neuro-cardiac model of human trunk development.

323 Our previous EMLO approach (Olmsted and Paluh, 2021a, 2021b) was developed to study early 324 neurogenesis events in trunk development. To investigate early neural lineage biomarkers in 325 EMLOCs we performed immunofluorescence and scRNAseq (Figure 8). TUJ1 immunostain was

326 first identified in cells opposite the anterior cardiac domain with low level staining, typical of neural 327 stem/progenitor cells that express this protein at lower levels (Figure 8A). The initial emergence 328 of neurons at day 7 , identified by morphology and biomarkers, parallels that seen for the original 329 EMLO protocol (Olmsted and Paluh, 2021a, 2021b). In EMLOCs, the posterior region of 330 neurogenesis emerged from one to several SOX2+/TUJ1+ neuroectodermal rosettes and the 331 number of neurons increased significantly over time (Figures 8B-8D). Given the relatively low 332 number of neurons present at the day 7 time point when spontaneous contractility is already 
333

334

335

336

337

338

339

340

341

342

343

occurring, it is unlikely that neuronal function plays a significant signaling role in initiating spontaneous cardiogenic contractions at this early stage. The increase in the number of TUJ1+ neurons in EMLOCs was quantified between days 7 and 18 (Figure 8E) (day 7: 5 +/- 2 TUJ1+ neurons, mean +/- s.e.m; day 18: $218+/-24$ TUJ1+ neurons; ${ }^{* * * *} \mathrm{p}<0.0001, \mathrm{t}=8.929$, df $=18$ by unpaired two-tailed t-test). We also quantified the proportion of EMLOCs with a neuronally integrated cardiac compartment between days 7 and 25 (Figure 8F), which similarly increased with time (day 7: $3.3+/-1.9 \%$ of population; day $25: 55.8+/-2.9 ;{ }^{* *} \mathrm{p}=0.0011, \mathrm{t}=12.58$, df $=3$ by paired two-tailed t-test; $N=4$ replicate experiments). A nidus of neurogenesis from neural rosettes occurred within GATA6+ surrounding mesenchymal-like tissue (Figure 8G). Notably, the gut tube can be distinguished from surrounding rosettes by a laminated acellular border, whereas the rosettes are more continuous with the adjacent GATA6+ cells (Figure S6). In EMLOCs, the open channels from the cardiac chambered region connect with the proximal compartment containing neural rosettes.

In the integrated scRNAseq dataset, clusters 1,8 and 10 predominantly represented the neural lineage. Neural progenitors (cluster 1; ZIC1, RFX4, HES5, FABP7, EDNRB, NTRK2, OLIG3, MSX1) became specialized neuronal subtypes (cluster 8; INSM1, ELAVL3, DLG4, CAMK2A, SLC18A3, SLC17A6, CHRNA3, NTRK3), and a population of neural crest-derived Schwann cells was present (cluster 10; SOX10, PLP1, MPZ, S100B, TFAP2B, NGFR) (Figures 1D-1F, Figure 8H). Combined INSM1/ISL1 expression indicates that sympathetic neurogenesis occurs in EMLOCs, which is particularly relevant to developing cardiac innervation. Restricted expression of HOXC6 and HOXC9 to these clusters supports spinal cord and trunk identity. Schwann cells increased $\sim 16$-fold from the day 7 to day 16 time point. As well, biomarkers of specialized neuronal subtypes that were lacking at day 7 began to emerge. The transcriptional phenotype for differentiating autonomic neurons that is ASCL1 (93/151 neurons, 62\%) and PHOX2B (14/151 neurons, $\sim 9 \%)$ predominated versus sensory neurons POU4F1/BRN3A (38/151 neurons, $\sim 25 \%$ ) or motor neurons MNX1/HB9 (6/151 neurons, $\sim 4 \%$ ) (Figure $8 \mathrm{H})$. This finding is distinct from EMLOs (Olmsted and Paluh, 2021a), in which motor neurons were primarily generated.

EMLOCs express biomarkers of cardiac innervation. In parallel with neurogenesis, axonal projections navigating the extracellular space to their target sites are expected to require spatial signals to generate the selective patterning on organs for innervation. Molecular and morphogenic features of the developing heart must therefore play an active role in establishing autonomic innervation, where the proper cellular milieu and receptive fields for innervation will dictate 
selective neuronal interactions. As such, we identified several genes with known roles in this process that were expressed in the cardiogenic region of the UMAP plot including and that code for neuropeptide $Y(N P Y)$, brain-derived neurotrophic factor (BDNF), semaphorin 3A (SEMA3A), peripherin $(P R P H)$, endothelin receptor type $\mathrm{A}(E D N R A)$, and ISL-1 (Figure 8I). Genes involved in autonomic neurogenesis and cardiogenesis such as ISL1 also play a role in development and

372 innervation of cardiac pacemaker cells that dictate automaticity and participate in the conduction 373 system apparatus.

By immunofluorescence, neurons were not identified within the cardiac region at the earlier day 7 and day 8 time points. We therefore analyzed the degree to which cardiogenic and neurogenic regions of the EMLOCs co-develop and integrate. In EMLOCs at day 16 or more in formation, neurons were observed both in the posterior compartment and intercalated with cardiomyocytes anteriorly, resembling in vivo ganglionated plexuses that characterize heart innervation (Ashton et al., 2018) (Figure S7). In day 25 EMLOCs (Figures 8J and 8K), the cardiogenic region remained contractile (Figure $\mathbf{8 J}$, Movie S5) and neurons formed elaborate structural networks within the myocardium that are excluded from the chambers, appreciated using 3D reconstruction analysis (Figure $\mathbf{8 K}$, Movie S6). Terminating neuronal fibers on cardiomyocytes were identified in part as axons using the phospho-tau (Ser214) immunostain (Figure S7E). We anticipate that the co-habitation of neurons and cardiomyocytes in the same region is a precursor to neuro-cardiac cooperative interactions such as initiation of innervation.

\section{DISCUSSION}

The consistent lifelong critical functioning of the adult human heart is established during embryonic development in a process known as cardiogenesis. EMLOCs provide the first detailed insights into integrated neurogenesis and cardiogenesis in a human gastruloid developmental model. The complex process of cardiogenesis requires short-range interactions with surrounding tissues and occurs in conjunction with long-range input by neurons through progressive innervation (Harvey, 2002; Hasan, 2013). As the first organ to function in the embryo, the developing heart begins to supply blood to the growing fetal brain as a closed tube, even before undergoing dramatic structural reorganization and maturation into septated chambers with

397 outflow tracts. Such complexity and dependence on multiple non-cardiac tissue inputs has made 398 it difficult to recapitulate human heart development using traditional in vitro models, requiring 399 instead refined gastruloid technologies. 
The intracardiac nervous system is sometimes colloquially referred to as the "brain within the heart" (Campos et al., 2018). Using sophisticated methodologies such as optogenetic stimulation, the role of peripheral cardiac neural circuitry in pacemaking and conduction is beginning to be understood (Rajendran et al., 2019; Fedele and Brand, 2020). Innervation of the heart in vivo is predominately autonomic, where sympathetic neurons can directly innervate working cardiomyocytes in the ventricular wall, and are networked as so-called ganglionated plexuses (Zaglia et al., 2017). The neurons that begin to develop in EMLOCs at the time when spontaneous contractility is first observed ( day 7 ) are unlikely to substantially contribute to contractile function, since at this stage they are relatively few in number and do not project into the cardiogenic region. This is consistent with in vivo development where contractility of the heart tube occurs prior to innervation that is established later (George et al., 2020). However, organized neuronal networks resembling ganglionated plexuses were observed as EMLOCs progressively matured. At the day 7 time point, neurons were localized distantly from the cardiogenic region, before expanding significantly in number to migrate, embrace and populate the myocardial layer over time. One potential explanation is a microenvironment switch from axon-repulsive to axonpermissive as ECM in the cardiac jelly is degraded. The ECM-rich cardiac jelly in vivo contains chondroitin sulfate proteoglycans and other components known to exert repulsive or pausing effects on axons during navigation and regeneration (Tom et al., 2004). Degradation of the cardiac jelly during development is physiologic and required for normal cardiac chamber morphogenesis (Kim et al., 2018). Differential regulation of SEMA3A expression may also play a role. Within this framework, our data support the adherence of EMLOC events to physiologic spatiotemporal developmental processes for establishing contractile chambers with supplied neurons (Hasan, 2013; George et al., 2020). Neuromuscular interactions between cardiac innervating neurons and cardiomyocytes at the "neuro-cardiac junction" remains poorly understood (Zaglia et al., 2017)

424 including biomarkers. Synapses with cardiomyocytes are postulated to be mediated through an 425 alternate structure other than the nAChR machinery in skeletal neuromuscular junctions (Sargent 426 and Garrett, 1995). Traditional 2D hiPSC differentiation protocols that generate human neurons 427 and cardiomyocytes separately and then co-culture these cells to obtain structural and functional 428 detail are typically used to study innervation, as has been done for the skeletal muscle 429 neuromuscular junction (Darabid et al., 2014; Steinbeck et al., 2016). EMLOCs are expected to 430 provide a developmental and spatiotemporal perspective of the neuro-cardiac junction.

Efforts to study heart development and function using human cells also focus on separate 432 cardiac mechanisms and include combined tissue engineering platforms and solutions (Ma et al., 433 2015; Macqueen et al., 2018). Such top-down human intervention of biofabricated tissues and 
434 organs has not yet achieved developmentally patterned neuronal innervation (Das et al., 2020),

435 but may benefit from this EMLOC study. Gastruloid models that more closely mimic 436 embryogenesis are an exciting alternative to achieve and study organogenesis (van den Brink et 437 al., 2014; Beccari et al., 2018; Moris et al., 2020). A recent study with mESCs made significant 438 advances and achieved early key features of cardiogenesis (Rossi et al., 2021). Our 439 developmental model of human cardiogenesis in gastruloids further advances cardiac models by 440 including neuronal co-development and association with the myocardium. The scRNAseq 441 analysis that indicates that we have established multiple prerequisites for innervation. In our 442 previous study of EMLO gastruloids that generated CNS and PNS integration with mixed lineage 443 trunk identity (Olmsted and Paluh, 2020a), we achieved self-organized spinal neurons, neural 444 crest, and a primitive gut tube surrounded by splanchnic mesenchyme, thereby providing much 445 of the ideal cardiogenic microenvironment. By modifying the EMLO protocol (Olmsted and Paluh, $4462020 \mathrm{~b}$ ) to include pro-cardiogenic and angiogenic factors, VEGF and ascorbic acid, that were 447 applied in the mESC in vitro cardiogenesis study (Rossi et al., 2021), we achieved coupled 448 cardiogenesis and neurogenesis. Through comprehensive biomarker analysis and live cell 449 calcium imaging, we demonstrate here that EMLOCs recapitulate numerous key features of 450 human cardiogenesis including cardiac crescent transformation into the contractile heart tube, 451 cardiomyocyte differentiation versus remodeling phases, and formation of chamber- and outflow 452 tract-like structures. Cardiogenesis occurs anterior to primitive gut tube-like endodermal cells that 453 in vivo are thought to be required (Nascone and Mercola, 1995; Schultheiss et al., 1995; Varner 454 and Taber, 2012; Anderson et al., 2016; Kidokoro et al., 2018; Han et al., 2020).

A limitation of this study is the use of one hiPSC line and ability to evaluate only two developmental time points by scRNAseq of the numerous stages analyzed and described. Nine 457 lines previously evaluated for EMLO formation revealed reproducibility of structural organization 458 and cell types but with differences in efficiency between lines that can be optimized (Olmsted and 459 Paluh, 2021a; 2021b). A second focus is expected to address how refined developmental insights 460 can be coupled with bioengineering technologies for development of 3D organogenesis platforms 461 bringing in additional features of vascularization. To understand the establishment and function 462 of the neuro-cardiac junction in EMLOCs, electrophysiologic and optogenetic characterization of 463 multi-tissue function beyond calcium imaging of neurons, cardiomyocytes, and neuro-cardiac 464 junction biomarkers is needed. Compared to the well-studied cholinergic neuromuscular junction 465 between motor neurons and skeletal muscle, much less is known about the autonomic synaptic 466 junctions between neurons and working cardiomyocytes including all relevant biomarkers 467 necessary to investigate innervation. Spatiotemporal analysis of scRNAseq data is further 
benefiting human developmental cell atlantes to complement existing databases for adult tissues (The Human Protein Atlas), and is being pursued for human cardiogenesis (Asp et al., 2019; Cui et al., 2019).

We anticipate that EMLOCs will open new opportunities to study fundamental questions

472 on neuromodulation of contracting cardiomyocytes with relevance to neurocardiogenic syncope

473 and other neural-based arrhythmia pathologies (Ashton et al., 2018). As well, such a neuro-

474 cardiac model system is expected to provide fundamental insights into the pathophysiology of

475 congenital heart disease and potential treatments in addition to viral infection studies and in vitro

476 pharmacotherapy testing and discovery. As a drastically needed component of in vitro stem cell

477 systems, innervation in non-neural tissue, organ, and embryo models (Das et al., 2020; Sahu and

478 Sharan, 2020) is beginning to be achieved in EMLO and EMLOC gastruloids to advance

479 innervation research. We expect that this approach and model will have broad biomedical relevance for neuro-cardiac development and human organ innervation initiatives.

In this work we develop and optimize a gastruloid model enabling co-development and selfintegration of human neuronal and cardiac tissue precursors in a multicellular, multi-lineage context. These results extend our previous work with neurogenesis and gut development in EMLO gastruloids to promote concomitant cardiogenesis that recapitulates multiple key features of in vivo heart development. In EMLOCs, neurons are produced endogenously in the context of the developing cardiac region as opposed to by separate differentiation and subsequent combination by fusion or in co-cultures. Genetic manipulation for optogenetic activation of neuro-cardiac pathways will inform on circuit formation and maturation. However, optical resolution in thick

492 samples with rapidly paced signaling and cell-cell communication events requires sophisticated 493 imaging techniques or further manipulation of the spatially organized cells such by slice cultures 494 that can help to dissect assembled pathways. Additional single cell sequencing at multiple 495 extended time points will be informative to clarify the diversity of cell types, transition states, and 496 signaling events. Future studies are expected to focus on continued developmental progression, 497 particularly addressing functional innervation, and the impact of these processes on diseases that 498 include ethnic contributions. EMLOCs therefore enable an exciting frontier to more rapidly 499 address neural innervation of non-neural structures in gastruloids that has remained a challenge 500 and priority of the field, and will continue to benefit from emerging technologies and resources. 


\section{ACKNOWLEDGEMENTS}

503 This work was funded by SUNY Polytechnic SEED 917035-21 and CATN2 MIP awards. It used 504 published lines developed and initially characterized through previous grants awarded to the 505 Paluh laboratory for New York State stem cell research (NYSTEM) and spinal cord injury 506 (NYSCIRB) research. Analysis by scRNAseq was performed at the SUNY Buffalo Genomics and 507 Bioinformatics core (SBGB). Jonathan Bard (SBGB) assisted in assembling the integrated 508 scRNAseq dataset and visualization methods by UMAP and PHATE for downstream analysis.

509

510 AUTHOR CONTRIBUTIONS

511 J.P. and Z.O. conceived of the project and experimental design and analyzed data and co-wrote 512 the manuscript. Z.O. performed EMLOC formation and characterization experiments, and 513 composed figures.

515 DECLARATION OF INTERESTS

516 A provisional USPTO patent on EMLOCs has been filed with patent application number $51763 / 311,498$. The authors declare no other competing interests.

520 Dr. Paluh received previous grant support (NYSTEM) to generate hiPSC lines with increased 521 ethnically diverse representation for basic and clinical science, including the human Hispanic 522 Latino iPSC line H3.1.1 used in this study. The authors have previously published several studies 523 that also apply hiPSC lines from self-reported African American, Hispanic Latino, and Asian 524 American fibroblast donors. 


\section{FIGURE LEGENDS}

Figure 1. EMLOC gastruloids capture cell type diversity of human cardiogenesis and trunk Immunofluorescence of day 7 H3.1.1 EMLOC immunostained for TUJ1 (red), cTnT (magenta), FOXA2 (cyan) and counterstained with DAPI (grey). Inset is high magnification Z-slice of FOXA2 foregut $(f g)$ initialization. Anterior $(A)$ to posterior $(P)$ orientation is descriptive. (C) 3D reconstruction of anterior cardiac region from (B) with TUJ1 (red), cTnT (magenta) and DAPI

537 (grey). The EMLOC chamber surface (left) and core (right) are shown. Individual scale bars 538 provided. (D) UMAP visualization of ten annotated clusters from the integrated scRNAseq dataset containing day 7 (1,004 cells) and day 16 (1,855 cells) time points in EMLOC formation (2,859 total cells). (E) PHATE visualization from the integrated dataset shown in (D). Cell lineages are labeled along with upregulated DEGs. (F) Heatmap of the top five DEGs for each cluster of the

542 integrated scRNAseq dataset (see also Table S1, Supplementary data file). Bright yellow

543 depicts upregulated differential gene expression. Abbreviations: anterior foregut (AFG), cardiac

544 fibroblast (CF), cardiomyocyte (CM), epicardial cells (EP), first heart field (FHF), genitourinary

545 (GU), intermediate mesoderm (IM), mitotic (mit), neuronal (N), outflow tract (OFT), second heart

546 field (SHF), splanchnic mesoderm (SM), vascular endothelial cells (VE).

Figure 2. Diverging lineages in EMLOCs advance according to in vivo developmental principles. (A) Day 16 EMLOC scRNAseq dataset visualized by PHATE. Superimposed developmental lineages include cardiac (TNNT2 or LHX9), anterior foregut (FOXA2 and NKX21), epithelial (PAX2 and PAX8), and neural (FABP7 or STMN2). (B) Signaling pathways segregate along diverging lineages in EMLOCs. BMP4 vs. BMP7 vs. SHH is shown (left) along with WNT2B vs. WNT1. (C) Preserved cadherin and Hox codes in EMLOCs. CDH1 vs. CDH6 vs.

$554 \mathrm{CDH11}$ is shown (left) along with HOXA4 vs. HOXC9 vs. HOXD8 (right). (D-E) Day 16 EMLOC scRNAseq dataset visualized by UMAP. (D) Genes for sarcomere proteins involved in contractility (TNNT2, TNNT1, MYL7) are upregulated primarily in cluster 3. Biomarkers for FHF (TBX5) and SHF (HAND2) are shown. (E) Epicardial cell and cardiac fibroblast biomarkers LHX2, LHX9, WT1 cluster 7 vs. cluster 2 . 
Figure 3. Spontaneous contractility and calcium handling in the EMLOC cardiac region. (A)

Sarcomeres are visible with cTnT at day 7 in EMLOC formation. Progressive zoom with high magnification black and white image (right). Yellow arrows depict striations. (B) Percentage of EMLOCs with beating chambers versus trunk biased gastruloids formed by the original EMLO protocol (Olmsted and Paluh, 2021a, 2021b). ( $N=3$ repeat experiments; ${ }^{* * *} \mathrm{p}=0.0005, \mathrm{t}=10.40$ $\mathrm{df}=4$ by unpaired two-tailed t-test). (C) Fluo-4 AM calcium imaging time course in two adjacent H3.1.1 EMLOCs. Individual scale bars provided. Images shown with fire LUT (ImageJ) and calibration bar. Individual scale bars provided. (D) Quantified F/Fo time series from (C). The two EMLOCs shown were captured in the same field and are representative of the population. (E) Box-and-whisker plot quantification of Fluo-4 AM Ca ${ }^{2+}$ transients/min in H3.1.1 day 7 EMLOCs ( $\mathrm{n}$ $=10$ EMLOCs, $\max =19, \min =5$, median $=10.5, \mathrm{q} 1=8, \mathrm{q} 3=12.75)$ from $\mathrm{N}=2$ separate differentiations. (F) Genes involved in rapid ventricular conduction (IRX3), repolarization (IRX5), calcium flux (ITPR2) and handling (SLC8A1/NAC1) are upregulated in EMLOCs. Day 16 scRNAseq data visualized by PHATE.

Figure 4. EMLOCs recapitulate early polarized heart tube formation events. (A) Cartoon schematic of the embryo depicting anterior cardiac crescent (cc, red/white), foregut cavity (fg, black), and neural tube (nt, dark blue). Anterior-posterior axis is indicated. The bilateral cardiac crescent fuses to form the transversal heart tube (HT), dorsally open heart tube, and linear heart tube (closed). (B) Percentage of day 4 gastruloids with cardiac crescent using the original EMLO protocol (Olmsted and Paluh, 2021a, 2021b) versus the optimized EMLOC protocol ( $\mathrm{N}=3$ repeat experiments; ${ }^{* *} \mathrm{p}=0.0007, \mathrm{t}=9.474$, df $=4$ by unpaired two-tailed $\mathrm{t}$-test). (C) Day 4 EMLOCs exhibit cTnT+ cardiac crescent-like structures (magenta, top) with GATA6+ nuclei (bottom, cyan). Phase image of day 4 EMLOC is shown with labeled $\mathrm{nt}$, $\mathrm{fg}$, and cc corresponding structures (top right). (D) Comparison of cTnT+ cardiogenic regions in day 4 versus day 5 EMLOCs. Two adjacent EMLOCs are shown for each time point. (E) High magnification of cardiac crescent structure with cTnT (magenta) and GATA6 (cyan). Individual channels are shown without pseudocolor. (F) Immunofluorescence of cTnT (magenta) and laminin (cyan) with inverted cTnT channels depicts developing heart tube-like structure in EMLOCs (day 6). Z-slices and cTnT maximal projections are shown. (G) Cardiac chamber precursors in two separate EMLOCs (day 7). Z-slices and cTnT maximal projections are shown. Individual scale bars provided.

593 Figure 5. Cardiogenesis occurs anterior to gut tube endoderm. (A) Left: cartoon schematic 594 of foregut and heart tube developmental cross-section with dorsal mesocardium (DM). The dorsal- 
ventral (D-V) and right-left (R-L) embryonic axes are shown. Right: cardiac crescent differentiation microenvironment. Cardiomyocyte progenitors (red), endocardium (tan), and definitive endoderm (blue) are shown. (B) Biomarker distribution using cTnT (cardiac, pink) and FOXA2 (gut tube, blue) in day 4 EMLOC gastruloids provided as smoothed curves. Normalized fluorescence was plotted over the anterior-posterior (A-P) axis normalized distance. (C) Left: Immunofluorescence Z-slices of FOXA2 (cyan)/Ki67 (magenta) depicts primitive foregut (fg) relative to cardiogenic region in day 5 versus day 6 EMLOC gastruloids. Right: CDH1/E-Cadherin depicts primitive gut tube (red) positioning relative to cTnT+ cardiogenic region (magenta). Cells are counterstained with DAPI. (D) Immunofluorescence Z-slices of GATA6 (cyan)/type 1 collagen (Col1, magenta) depicts primitive gut tube relative to cardiogenic region during chamber precursor formation in day 5 (left) versus day 6 (right) EMLOC gastruloids. (E) CTnT (magenta) and CDH2/N-Cadherin (cyan) co-localization in cardiac crescent reveals epithelization of cardiomyocyte progenitors. Inset depicts cTnT+ without pseudocolor. Yellow arrows depict striations. High magnification images with and without DAPI are provided (right) at the boundary of the EMLOC cardiogenic region. Individual scale bars provided.

Figure 6. Myocardial expansion and heart tube morphogenetic specialization. (A) Cartoon schematic of cardiogenesis from cardiac crescent to heart tube specialization and heart tube looping. Truncus arteriosus (TA), sinus venosus (SV). (B) Heart tube staging in day 7 EMLOC gastruloids by cTnT (magenta) and TUJ1 (red), corresponding to the boxed stage in (A). Maximally projected Z-stacks and single Z-slice is shown. (C) 3D reconstruction of the anterior cardiac region with cTnT (magenta) and DAPI (grey) depicting putative outflow tract (yellow arrows) and chambers in two EMLOC gastruloids. (D) Multi-dimensional visualization of cTnT

618 (magenta) and DAPI (grey). Sagittal and transverse planes are shown. Individual scale bars 619 provided. Asterisks $\left(^{*}\right)$ indicate communication with proximal EMLOC compartment. Individual 620 scale bars provided.

622 Figure 7. Chamber precursor morphogenesis in EMLOCs. (A) Cartoon schematic of a 623 developing heart tube. Chamber wall layers are expanded to depict myocardium, extracellular 624 matrix-rich cardiac jelly, and endocardium interior lining. (B) 3D reconstruction of the anterior 625 cardiac chamber-like structures with cTnT+ myocardium (magenta) and Laminin+ interior (top626 left). Single Z-slice multi-dimensional view of chamber (top-right). High magnification images are 627 provided below. (C) VCAN, ADAMTS1, ANGPT1 genes involved in cardiac jelly and its 628 spatiotemporal degradation in day 16 EMLOC by scRNAseq, visualized using PHATE. (D) 
629 Immunofluorescence of cTnT, GATA4 and DAPI demonstrating putative outflow tract (white

630 arrows) in two day 7 EMLOCs. Z-slice inset provides a second example. (E) Immunofluorescence

631 of N-Cadherin (CDH2, cyan), VE-Cadherin (vascular endothelial cadherin/CD144/CDH5,

632 magenta), and DAPI (grey) depicting endothelial biomarker expression lining the putative outflow

633 tract. Rightmost zoom image depicts valve-like crescent structures. Individual scale bars

634 provided. (F) Biomarkers for smooth muscle cells (CNN1/TAGLN) and the outflow tract

635 (ISL1/PDE5A/CDH11) along with vascular endothelial cells (KDR/FLT1/ESAM/CDH5) (cluster 9).

636 (G) POSTN, TBX3, NPR3, NFATC4 genes involved in atrioventricular valve formation.

Figure 8. Neuron co-development and population of the cardiac region. (A) Neural rosette biomarkers SOX2 (cyan) and TUJ1 (red) emerging posteriorly in day 7 H3.1.1 EMLOC, counterstained with DAPI. (B, C) SOX2+ rosette and neurogenesis (TUJ1) counterstained with DAPI along with high magnification (C). Inset is whole EMLOC. (D) Immunofluorescence of cTnT (cyan) and TUJ1 (red) depicting single neuron in day 8 EMLOC (left, white dotted box) and zoom in (middle panel). Comparison with TUJ1+ neuron tract in day $18 \mathrm{EMLOC} \mathrm{(far} \mathrm{right} \mathrm{panel).} \mathrm{(E)}$ Absolute number of TUJ1+ cells in day 7 versus day 18 EMLOCs ( $n=10$ per time point). ${ }^{* * *} p<$ $0.0001, \mathrm{t}=8.929, \mathrm{df}=18$ by unpaired two-tailed t-test. $(\mathbf{F})$ Proportion of EMLOCs with TUJ1+ neuronal fibers distributed within the cardiac region at day 7 versus day $18(\mathrm{~N}=4$ replicate experiments; day $7: 3.3+/-1.9 \%$ of population; day $25: 55.8+/-2.9 ;{ }^{* *} p=0.0011, t=12.58$, df $=$ 3 by paired two-tailed t-test). (G) Nidus of neurogenesis posterior to gut tube endodermal cells and cardiogenic region in day 8 EMLOC by TUJ1 (red), GATA6 (cyan), and cTnT (magenta). Maximally projected Z-stack (Z-total) and single Z-slice shown. White arrows point to communicating channels. Gut tube endoderm is laminated while neural rosettes are continuous with surrounding GATA6+ cells. (H) Left: UMAP representation of day 16 EMLOC scRNAseq highlighting trunk neural progenitors (ZIC1/RFX4), sympathetic neurogenesis (INSM1/ISL1) and Schwann cell glia (SOX10/PLP1). Right: quantification of neuronal class proportions in clusters 1, 8, 10 as autonomic (ASCL1 62\%, PHOX2B 9\%), sensory (POU4F1 25\%) and motor (MNX1 $4 \%$ ). (I) Genes in the cardiogenic region involved in neuronal patterning and innervation. NPY, BDNF, SEMA3A, PRPH, EDNRA, ISL1 shown by UMAP (day 16). (J) Phase contrast image of contractile EMLOC chamber-like structures (see also Movie S5). (K) 3D reconstruction of TUJ1+ neuronal fiber intercalation with chamber-like myocardium in day 25 EMLOC (see also Movie S6). Rotated view is shown. Individual scale bars provided. 


\section{SUPPLEMENTAL MOVIES}

665 Movie S1. Contractile EMLOCs in suspension culture at day 7. Phase contrast real-time

666 movie at 5x magnification. Related to Figure S1.

668 Movie S2. Contractile EMLOCs in suspension culture at day 7. Phase contrast real-time 669 movie at 20x magnification. Related to Figure S1.

671 Movie S3. Live cell calcium imaging for contractility with whole EMLOCs at day 7. Example 6721 with Fluo-4 AM dye. The cardiogenic regions of the two EMLOCs are adjacent. 200 ms interval, 67350 ms exposure time, 17 frames per second. Related to Figures 3C-3E.

674

675 Movie S4. Live cell calcium imaging for contractility with whole EMLOCs at day 7. Example

6762 with Fluo-4 AM dye. Calcium fluorescence correlates with visible contractility. 200 ms interval,

$67750 \mathrm{~ms}$ exposure time, 17 frames per second. Related to Figures 3C-3E.

678

679 Movie S5. Contractile EMLOCs in suspension culture at day 25. Phase contrast real-time 680 movie at 20x magnification. Related to Figure 8J.

681

682 Movie S6. 3D reconstruction of innervated cardiac chambers. Generated using Imaris. 683 Related to Figure 8K. 


\section{METHODS}

687

\section{RESOURCE AVAILABILITY}

\section{Lead contact}

690 Further information and reasonable requests for resources should be directed to and will be

691 fulfilled by the Lead Contact, Janet L. Paluh (paluhj@sunypoly.edu).

692

\section{Materials availability}

694 This study did not generate unique reagents.

695

696

\section{Data and code availability}

697 The scRNAseq data is available through GEO (GSE194356). The authors declare that all other 698 data supporting the findings of this study are available within the article and its Supplementary 699 Information files or from the corresponding author upon reasonable request.

700

\section{KEY RESOURCES TABLE}

\begin{tabular}{|l|l|l|}
\hline REAGENT or RESOURCE & SOURCE & IDENTIFIER \\
\hline Antibodies & R\&D Systems & Cat.No.: AF2018; RRID: AB_355110 \\
\hline Goat anti-SOX2 & R\&D Systems & Cat.No.: AF2606; RRID: AB_2232177 \\
\hline Goat anti-GATA4 & R\&D Systems & Cat.No.: AF1700; RRID: AB_2108901 \\
\hline Goat anti-GATA6 & $\begin{array}{l}\text { Novus } \\
\text { Biologicals }\end{array}$ & Cat.No.: NBP1-30054; RRID: AB_1968486 \\
\hline Rabbit anti-Collagen I $\alpha 1$ & $\begin{array}{l}\text { Novus } \\
\text { Biologicals }\end{array}$ & Cat.No.: NB300-144 \\
\hline Rabbit anti-Laminin & R\&D Systems & Cat. No.: AF648; RRID: AB_355504 \\
\hline Goat anti-CDH1/E-cadherin & $\begin{array}{l}\text { Cell Signaling } \\
\text { Technologies }\end{array}$ & Cat.No.: 13116; RRID: AB_2687616 \\
\hline Rabbit anti-CDH2/N-cadherin & R\&D Systems & Cat. No.: MAB9381; RRID: AB_2260374 \\
\hline $\begin{array}{l}\text { Mouse anti-VE } \\
\text { Cadherin/CDH5/CD144 }\end{array}$ & R\&D Systems & Cat.No.: AF2400; RRID: AB_2294104 \\
\hline Goat anti-FOXA2 & BioLegend & Cat.No.: 802001; RRID: AB_2564645 \\
\hline $\begin{array}{l}\text { Rabbit anti-B-tubulin III/TUJ1 } \\
\text { (TUBB3) }\end{array}$ & Invitrogen & Cat.No.: 44-742G; RRID: AB_2533740 \\
\hline Rabbit anti-Phospho Tau (Ser214) & \\
\hline
\end{tabular}




\begin{tabular}{|c|c|c|}
\hline Goat anti-Desmin & R\&D Systems & Cat.No.: AF3844; RRID: AB_2092419 \\
\hline Mouse anti-cardiac Troponin T & R\&D Systems & Cat.No.: MAB1874; RRID: AB_2206731 \\
\hline \multicolumn{3}{|c|}{ Chemicals, peptides and recombinant proteins } \\
\hline mTeSR Plus & $\begin{array}{l}\text { STEMCELL } \\
\text { Technologies }\end{array}$ & Cat.No.: 05825 \\
\hline mFreSR & $\begin{array}{l}\text { STEMCELL } \\
\text { Technologies }\end{array}$ & Cat.No.: 05854 \\
\hline DMEM/F-12 & $\begin{array}{l}\text { Thermo Fisher } \\
\text { Scientific }\end{array}$ & Cat.No.: 11320033 \\
\hline Neurobasal Plus Medium & $\begin{array}{l}\text { Thermo Fisher } \\
\text { Scientific }\end{array}$ & Cat.No.: A3582901 \\
\hline $\begin{array}{l}\text { BrainPhys Neuronal Culture } \\
\text { Medium }\end{array}$ & $\begin{array}{l}\text { STEMCELL } \\
\text { Technologies }\end{array}$ & Cat.No.: 05790 \\
\hline N-2 supplement (100X) & $\begin{array}{l}\text { Thermo Fisher } \\
\text { Scientific }\end{array}$ & Cat.No.: 17502048 \\
\hline B-27 supplement (50X) & $\begin{array}{l}\text { Thermo Fisher } \\
\text { Scientific }\end{array}$ & Cat.No.: 17504044 \\
\hline GlutaMAX & $\begin{array}{l}\text { Thermo Fisher } \\
\text { Scientific }\end{array}$ & Cat.No.: 35050061 \\
\hline MEM Non-Essential Amino Acids & $\begin{array}{l}\text { Thermo Fisher } \\
\text { Scientific }\end{array}$ & Cat.No.: 11140050 \\
\hline Penicillin-Streptomycin & $\begin{array}{l}\text { Thermo Fisher } \\
\text { Scientific }\end{array}$ & Cat.No.: 15140122 \\
\hline hESC-qualified Matrigel & Corning & Cat.No.: 08-774-552 \\
\hline CHIR 99021 & $\begin{array}{l}\text { Tocris } \\
\text { Bioscience }\end{array}$ & Cat.No.: 4423 \\
\hline bFGF/FGF2 & R\&D Systems & Cat.No.: 233-FB \\
\hline HGF & R\&D Systems & Cat.No.: 294-HG \\
\hline IGF-1 & R\&D Systems & Cat.No.: 291-G1 \\
\hline VEGF 165 & R\&D Systems & Cat.No.: 293-VE \\
\hline L-ascorbic acid & Tocris & Cat.No.: 4055 \\
\hline $\mathrm{Y}-27632$ & $\begin{array}{l}\text { Tocris } \\
\text { Bioscience }\end{array}$ & Cat.No.: 1254 \\
\hline Accutase & $\begin{array}{l}\text { STEMCELL } \\
\text { Technologies }\end{array}$ & Cat.No.: 07920 \\
\hline Gentle Cell Dissociation Reagent & $\begin{array}{l}\text { STEMCELL } \\
\text { Technologies }\end{array}$ & Cat.No.: 07174 \\
\hline
\end{tabular}




\begin{tabular}{|c|c|c|}
\hline Anti-Adherence Rinsing Solution & $\begin{array}{l}\text { STEMCELL } \\
\text { Technologies }\end{array}$ & Cat.No.: 07010 \\
\hline Bacillus Licheniformis protease & $\begin{array}{l}\text { Creative } \\
\text { Biomart }\end{array}$ & Cat.No.: NATE-0633 \\
\hline DNase I & Sigma-Aldrich & Cat.No.: 11284932001 \\
\hline $\begin{array}{l}\text { CryoStor CS-10 for } \\
\text { cryopreservation (scRNAseq } \\
\text { shipping) }\end{array}$ & $\begin{array}{l}\text { BioLife } \\
\text { Solutions }\end{array}$ & Cat.No.: 210373 \\
\hline Fetal bovine serum & $\begin{array}{l}\text { Thermo Fisher } \\
\text { Scientific }\end{array}$ & Cat.No.: 26140087 \\
\hline Fluo-4 AM & $\begin{array}{l}\text { Thermo Fisher } \\
\text { Scientific }\end{array}$ & Cat.No.: F14201 \\
\hline HBSS & $\begin{array}{l}\text { Thermo Fisher } \\
\text { Scientific }\end{array}$ & Cat.No.: 14025076 \\
\hline HBSS CM-free & $\begin{array}{l}\text { Thermo Fisher } \\
\text { Scientific }\end{array}$ & Cat.No.: 14175079 \\
\hline Histodenz & Sigma-Aldrich & Cat.No.: D2158 \\
\hline Bovine Serum Albumin Fraction V & Fisher Scientific & Cat.No.: BP1600-100 \\
\hline Triton $\mathrm{X}-100$ & $\begin{array}{l}\text { Electron } \\
\text { Microscopy } \\
\text { Sciences }\end{array}$ & Cat.No.: 221440 \\
\hline NucBlue fixed cell stain & $\begin{array}{l}\text { Thermo Fisher } \\
\text { Scientific }\end{array}$ & Cat.No.: R37606 \\
\hline \multicolumn{3}{|l|}{ Experimental models: Cell lines } \\
\hline H3.1.1 & Paluh lab & Chang et al., 2015; Tomov et al., 2016 \\
\hline \multicolumn{3}{|l|}{ Software and algorithms } \\
\hline Keynote & Apple & https://www.apple.com/keynote/ \\
\hline GraphPad Prism 9 & GraphPad & $\begin{array}{l}\text { https://www.graphpad.com/scientific- } \\
\text { software/prism/ }\end{array}$ \\
\hline Excel & Microsoft & $\begin{array}{l}\text { https://www.microsoft.com/en-us/microsoft- } \\
\text { 365/excel }\end{array}$ \\
\hline Fiji & $\begin{array}{l}\text { Schindelin et } \\
\text { al., } 2012\end{array}$ & https://imagej.net/Fiji \\
\hline Illustrator CC2020 & Adobe & https://www.adobe.com/products/illustrator.html \\
\hline Imaris & BitPlane & https://imaris.oxinst.com/ \\
\hline cellSens & Olympus & $\begin{array}{l}\text { https://www.olympus- } \\
\text { lifescience.com/en/software/cellsens/ }\end{array}$ \\
\hline Cell Ranger & 10x Genomics & https://www.10xgenomics.com \\
\hline
\end{tabular}




\begin{tabular}{|l|l|l|}
\hline Seurat & Satija lab & https://github.com/satijalab/seurat \\
\hline PHATE & $\begin{array}{l}\text { Krishnaswamy } \\
\text { lab }\end{array}$ & https://github.com/KrishnaswamyLab/PHATE \\
\hline Loupe & $10 x$ Genomics & https://www.10xgenomics.com \\
\hline Other & CELLTREAT & Cat.No.: 229105 \\
\hline 6 well plate & Fisher & Cat.No.: S33580A \\
\hline 100 mm petri dish & lbidi & Cat.No.: 80826 \\
\hline$\mu$-slide 8 well & $\begin{array}{l}\text { Chemglass Life } \\
\text { Sciences }\end{array}$ & Cat.No.: CLS-4021-100 \\
\hline $\begin{array}{l}\text { CHEMcell VERSA-ORB2 orbital } \\
\text { shaker }\end{array}$ &
\end{tabular}

\section{EXPERIMENTAL MODELS AND SUBJECT DETAILS}

704 Human induced pluripotent stem cells. We previously derived hiPSC lines from Coriell deidentified human fibroblast samples from consenting donors, including the Hispanic-Latino H3.1.1 line used in this study (Chang et al., 2015; Tomov et al 2016). Line H3.1.1 was reprogrammed with Yamanaka factors by the laboratories of Dr. Paluh and Dr. Jose Cibelli from these donor fibroblasts and comprehensively characterized for pluripotency (immunofluorescence, RT-PCR), G-band karyotype, teratoma formation, multi-lineage differentiation, bulk RNA-Seq, ChIP-Seq, and used in multiple studies from this lab. Recent G-band karyotype validation and pathogen analysis was performed by Cell Line Genetics, Inc. (NY, NY). H3.1.1 hiPSC colonies were

712 cultured in mTeSR Plus supplemented with 1x penicillin-streptomycin (P-S) on hESC-qualified

713 Matrigel ( $1: 100$ dilution; Corning) in a humidified incubator at $37^{\circ} \mathrm{C}, 5 \% \mathrm{CO}_{2}$. Cultures were 714 passaged 1:6 in 6-well plates every 4-7 days using Gentle Cell Dissociation Reagent (GCDR, 715 STEMCELL Technologies). Cells were cryopreserved in mFreSR.

\section{METHOD DETAILS}

718 EMLOC formation. EMLOs were formed similarly to as previously described (Olmsted and Paluh, 719 2021a, 2021b) with several important differences detailed as follows. H3.1.1 adherent hiPSC 720 colonies were maintained in mTeSR Plus pluripotency medium as described above. At $\sim 60 \%$ 721 confluency, pluripotency medium was changed to induction medium (N2B27 basal medium 722 supplemented with $3 \mu \mathrm{M}$ CHIR 99021, $40 \mathrm{ng} / \mathrm{ml}$ basic fibroblast growth factor FGF2). N2B27 723 basal medium: 1:1 DMEM/F-12:Neurobasal Plus medium, 2\% (v/v) B27 Plus supplement, 1\% 724 (v/v) N2 supplement, 1x GlutaMAX, 1x MEM Non-Essential Amino Acids, 1x P-S. Adherent hiPSC 
colonies were induced for two days the one exchange of fresh medium at $24 \mathrm{~h}$. On the day of aggregation, cells were dissociated with 1:1 Accutase:HBSS (Ca-Mg free) at $37^{\circ} \mathrm{C}$ for $5 \mathrm{~min}$

727 followed by manual trituration with a P-1000 pipette. Six-well plates were pre-treated with Anti728 Adherence Rinsing Solution (STEMCELL Technologies) for 5 min incubation at room temperature 729 followed by two rinses with equal volumes of HBSS. Cells were resuspended in N2B27 730 supplemented with $10 \mathrm{ng} / \mathrm{ml}$ FGF2, 2 ng/ml IGF-1, 2 ng/ml HGF (R\&D Systems) and $50 \mu \mathrm{M} \mathrm{Y-}$ 73127632 (Tocris Bioscience). For aggregation, the single cell suspensions were added at a density 732 of $2 \times 10^{6}$ cells $/ \mathrm{ml}$ ( $2 \mathrm{ml}$ per well, $4 \times 10^{6}$ total cells). Gastruloids were aggregated overnight using 733 an orbital shaker at $75 \mathrm{rpm}$ clockwise in a humidified incubator with $5 \% \mathrm{CO}_{2}$. The next day, one734 half volume of medium was replaced with fresh medium N2B27 supplemented with $4 \mathrm{ng} / \mathrm{ml}$ IGF$7351,4 \mathrm{ng} / \mathrm{ml} \mathrm{HGF}, 20 \mathrm{ng} / \mathrm{ml} \mathrm{FGF2}$ to maintain the same concentration of growth factors in the culture 736 medium after one-half volume addition. At $48 \mathrm{~h}$, the entire volume of medium was replaced with 737 N2B27 basal medium supplemented with $5 \mathrm{ng} / \mathrm{ml} \mathrm{VEGF,} 30 \mathrm{ng} / \mathrm{ml}$ FGF2, and $0.5 \mathrm{mM}$ ascorbic 738 acid (Rossi et al., 2021). EMLOCs were induced in this medium to day 5. At day 7, the EMLOCs were maintained in non-supplemented N2B27. For orbital shaking culture, cells were aggregated and induced at $80 \mathrm{rpm}$. Speed was reduced to $75 \mathrm{rpm}$ on day 7.

EMLOC single-cell dissociation by cold activated protease for scRNAseq. Type here (UB)

743 Day 7 and Day 16 EMLOCs were dissociated on their respective time points in differentiation 744 according to the protocol we previously described (Olmsted and Paluh, 2021a). In brief, 25 745 EMLOCs from each time point were pooled in a $2 \mathrm{ml}$ centrifuge tube and exposed to $1 \mathrm{ml}$ 746 dissociation solution composed of $10 \mathrm{mg} / \mathrm{ml}$ Bacillus licheniformis protease and $125 \mathrm{U} / \mathrm{ml} \mathrm{DNase}$ 747 in ice-cold 1x PBS supplemented with $5 \mathrm{mM}$ calcium chloride. EMLOCs were incubated on ice in 748 dissociation solution and triturated with a P-1000 pipette every 30-60 s for 8 min. Dissociation to 749 single cells was verified by optical inspection and the reaction was terminated by addition of $1 \mathrm{ml}$ 750 ice-cold 1x PBS with 10\% fetal bovine serum (FBS). Cells were pelleted by centrifugation at 1,200 $751 \times g$ for $5 \mathrm{~min}$, resuspended in fresh 1x PBS/10\% FBS, counted, and centrifuged once more.

752 Supernatant was aspirated completely and cells were resuspended in CryoStor CS10 753 cryopreservation medium to a final concentration of $1 \times 10^{6}$ cells per $\mathrm{ml}$, filtered through a $40 \mu \mathrm{m}$ 754 cell strainer, and transferred to a $1.8 \mathrm{ml}$ Nunc cryo-storage tube. Cells were frozen at $-80^{\circ} \mathrm{C}$ 755 overnight and transferred to a liquid nitrogen dewar. When samples from both time points were 756 dissociated and stored, samples were shipped overnight on dry ice to University of Buffalo 757 Genomics and Bioinformatics Core at the New York State Center of Excellence in Bioinformatics 758 and Life Sciences. 
Single-cell sequencing with CellPlex, cluster annotation and analysis. When samples were received, they were immediately stored at $-80^{\circ} \mathrm{C}$. On the day of cell capture for sequencing, day 7 and day 16 EMLOC samples were thawed in a $37^{\circ} \mathrm{C}$ water bath. Individual time point samples were transferred to separate $15 \mathrm{ml}$ tubes. RPMI1640+10\% FBS pre-warmed media was added dropwise to a final volume of $10 \mathrm{ml}$ per tube. Cells were centrifuged at $300 \times g$ for $5 \mathrm{~min}$. This washing procedure was performed a total of three times. After the final wash, medium was completely removed and cell samples were separately resuspended in $100 \mu \mathrm{l}$ of Cell Multiplexing Oligo (10x Genomics). The two populations were suspended with two different oligos as directed by the manufacturer's instructions. After a brief incubation, cells were washed $3 x$ with ice cold $1 x$ PBS $(\mathrm{pH} \mathrm{7.4)}+1 \%$ bovine serum albumin (BSA). Cells were then resuspended in $250 \mathrm{ul} 1 \mathrm{x}$ PBS $/ 1 \%$ BSA and counted on a Logos Biosystems LUNA II in bright field mode with $0.4 \%$ trypan blue. The two cell populations with different barcodes were then pooled to 10,000 cells $(5,000$ from each time point) and recounted. The combined single cell suspension was provided as input for the 10x Genomics Single Cell v3.1 protocol with Feature Barcode technology. After libraries were prepared, they were loaded onto an Illumina NextSeq in high-output mode with a general target of 50,000 reads per cell to provide for sufficient depth and transcriptomic saturation. Post sequencing, data was demultiplexed and provided as input into the 10x Genomics Cell Ranger multipipeline (ver 4), which quantifies the transcriptomic profile of each cell against a reference genome. Sequence saturation, detected barcodes per cell, percent of transcripts in cell, and general alignment statistics were evaluated for quality. Cell Ranger matrix files were then used as input into the R Bioconductor package Seurat (ver 4). Cells with outlier-status, abnormal gene detection rates, and high mitochondrial transcript load that is an indicator of cellular stress were filtered from the analysis. After filtering, the data was underwent Seurat normalization and integrated using the SCTransform and integration protocol, followed by UMAP (Uniform Manifold Approximation and Projection) or the alternative PHATE dimensionality reduction for visualization (Moon et al., 2019). Using the called clusters, cluster-to-cluster differential expression testing using the Wilcoxon Rank Sum Test was used to identify cluster-defining biomarkers, as well as further exploratory analysis with known biomarker genes (gene lists provided in Supplementary data file). Data was then exported from Seurat for further analysis in the Loupe browser.

790 Calcium imaging of contractility. The EMLOCs were incubated with Fluo-4 AM dye as 791 described above in $1 \mathrm{ml}$ of medium for $30 \mathrm{~min}$. Cells were rinsed once in HBSS and imaged in 792 BrainPhys medium without Phenol red. Timelapse series were acquired at 50 ms exposure using 
a $488 \mathrm{~nm}$ LED at $200 \mathrm{~ms}$ intervals for $1.5 \mathrm{~min}$ duration. Analysis of calcium spike transients was performed using ImageJ. We performed wide field fluorescence microscopy using a Zeiss Axio Observer.Z1 inverted fluorescence microscope (20x/0.8 air objective for live cell calcium imaging). Images were acquired using an Hamamatsu ORCA ER CCD camera and Zeiss AxiovisionRel

797 software (ver. 4.8.2).

Phase contrast and whole mount immunofluorescence. Phase contrast microscopy was performed at room temperature directly in the biosafety hood. Images were acquired using a Zeiss Invertoskop 40C (5x/0.12 CP-Apochromat, 10x/0.25 Ph1 A-Plan and 20x/0.30 Ph1 LD A-Plan, 40x/0.50 Ph2 LD A-Plan) mounted with an Olympus DP22 color camera and cellSens acquisition software. Whole-mount immunofluorescence preparation was performed as previously described (Veenvliet et al., 2020; Olmsted and Paluh, 2021). EMLOCs were pooled on the day of fixation, rinsed once with 1x phosphate-buffered saline (PBS), and fixed in 10\% neutral buffered formalin solution at $4^{\circ} \mathrm{C}$ for $2 \mathrm{~h}$. Samples were washed three times in $1 \mathrm{x}$ PBS for $5 \mathrm{~min}$ at room temperature. Samples were then permeabilized by three successive incubations in $0.2 \%$ Triton $\mathrm{X}-100$ in 1x PBS (PBST) for 20 min at $4^{\circ} \mathrm{C}$, and blocked overnight in 1\% BSA in PBST. For primary antibody incubation, samples were distributed evenly to 12-well plates in $1 \mathrm{ml}$ blocking solution per well. Primary antibodies were added to requisite dilutions in 1\% BSA (1x PBS): anti-SOX2 (goat, $5 \mu \mathrm{g} / \mathrm{ml}$ ); anti-GATA4 (5 $\mu \mathrm{g} / \mathrm{ml})$; anti-GATA6 $(5 \mu \mathrm{g} / \mathrm{ml})$; anti-CDH1/E-Cadherin $(5 \mu \mathrm{g} / \mathrm{ml})$;

812 anti-FOXA2 (5 $\mu \mathrm{g} / \mathrm{ml})$; anti- $\beta$-IIl-tubulin (rabbit, 1:2,000); phospho-Tau Ser214 (rabbit, $1 \mu \mathrm{g} / \mathrm{ml}$ ); $813 \mathrm{CDH} / \mathrm{N}-$ Cadherin (1:200); anti-Collagen Type 1 (1:500, $1 \mathrm{mg} / \mathrm{ml}$ stock); anti-Laminin (1:500, 1 $814 \mathrm{mg} / \mathrm{ml})$; anti-Desmin $(5 \mu \mathrm{g} / \mathrm{ml})$; anti-Cardiac Troponin-T $(25 \mu \mathrm{g} / \mathrm{ml})$. Plates were left rocking at $4^{\circ} \mathrm{C}$ 815 for 24-48 h, rinsed three times in blocking solution, then three times in PBST for 5 min each at room temperature ( $2 \mathrm{ml}$ centrifuge tubes). Secondary antibodies were incubated 1:1,000 with 2 drops of NucBlue fixed cell stain (Invitrogen) directly in the $2 \mathrm{ml}$ tubes overnight at $4^{\circ} \mathrm{C}$. Goat antimouse Cy5 secondary antibody was added the next day following washes steps to dilute donkey anti-goat AlexaFluor 594 secondary antibody for samples stained with three antibodies (mouse, rabbit, goat). Samples were again incubated overnight rocking at $4^{\circ} \mathrm{C}$. Stained and rinsed EMLO

821 samples were post-fixed in $10 \%$ neutral buffered formalin for 20 min at $4^{\circ} \mathrm{C}$, and equilibrated in $8220.1 \mathrm{M}$ phosphate buffer (PB: $0.025 \mathrm{M} \mathrm{NaH}_{2} \mathrm{PO}_{4}, 0.075 \mathrm{M} \mathrm{Na}_{2} \mathrm{HPO}_{4}, \mathrm{pH} 7.4$ ) containing $0.2 \%$ 823 Triton-X 100 by three successive incubations of $5 \mathrm{~min}$ at room temperature. To clear samples, 8240.1 M PB was aspirated and replaced with $100 \mu \mathrm{l}$ of $88 \%$ Histodenz solution (w/v) dissolved in $0.2 \mathrm{M}$ PB and filter sterilized. Samples were left in the dark at $4^{\circ} \mathrm{C}$ for $24 \mathrm{~h}$, mounted on glass slides and sealed in clear nail polish for imaging. Samples were imaged on a Leica confocal TCS 
827 SP5 II system in conjunction with Leica Application Suite Advanced Fluorescence software. The

828 SP5 II system was equipped with 10x/0.30 HCX PL FLUOTAR air, 20x/0.70 HC PL APO CS air

829 or immersion, and 40x/1.25 HCX PL APO immersion objective lenses. Complete or partial Z-

830 stacks were acquired at $\sim 2-2.5 \mu \mathrm{m}$ separation distance. If necessary, images were corrected

831 linearly for brightness in ImageJ. Maximally projected Z-stacks were performed directly in the

832 Leica software and exported, or were made using Z-project in ImageJ.

\section{QUANTIFICATION AND STATISTICAL ANALYSIS}

835 Quantification of immunofluorescence signal. Quantification immunofluorescence signals 836 along the anterior-posterior axis was performed as described (Rossi et al., 2021). cTnT signal 837 was quantified from maximal projection images (z-axis) and the FOXA2 was quantified from single 838 Z-slices in order to capture the gut tube. The anterior-posterior axis length was measured from 839 pole-to-pole for each gastruloid. Fluorescence intensity was determined using the plot profile tool 840 in Fiji ImageJ, and was normalized along with gastruloid length to enable comparative analysis. 841 FOXA2 and cTnT mean curves were plotted in GraphPad Prism 9 and juxtaposed. Curves were 842 smoothed using a LOWESS function in GraphPad. Only the single channels in question were 843 quantified.

845 Statistical analysis and reproducibility. Microsoft Excel (v16.16.27) and GraphPad Prism 9 846 (v9.0.2) were used for statistical analysis and data plotting. Data are reported as (mean +/847 s.e.m.), analyzed using paired or unpaired two-tailed t-test as indicated. ${ }^{* * *} p<0.0001,{ }^{* * *} p<0.001$, $848{ }^{* *} p<0.01,{ }^{*} p<0.05$, n.s. not significant $(\alpha=0.05$ threshold for significance). Power analysis was not 849 performed. Detailed information for each experiment is provided in Results and Figure Legends. 850 Key resources including primary antibodies, chemicals and other reagents, software and 851 equipment, and commercial kits are provided (Key Resources Table above).

853 Figures. Figures for this manuscript were made with a combination of Keynote (v10.3.8), Adobe 854 Illustrator Creative Commons 2020, and BioRender.com. Data were analyzed using Excel and 855 GraphPad Prism 9, and plots were generated using GraphPad Prism 9. 


\section{REFERENCES}

Andersen, P., Tampakakis, E., Jimenez, D. V., Kannan, S., Miyamoto, M., Shin, H. K., Saberi, A., Murphy, S., Sulistio, E., Chelko, S. P., et al. (2018). Precardiac organoids form two heart fields via $\mathrm{Bmp} /$ Wnt signaling. Nat. Commun. 9, 3140.

Anderson, C., Khan, M.A.F., Wong, F., Solovieva, T., Oliveira, N. M. M., Baldock, R. A. et al. (2016). A strategy to discover new organizers identifies a putative heart organizer. Nat. Commun. 7, 12656.

Ashton, J.L., Burton, R.A.B., Bub, G., Smaill, B.H., Montgomery, J.M. 2018. Synaptic plasticity in cardiac innervation and its potential role in atrial fibrillation. Front. Physiol. 9, 240.

Asp, M., Giacomello, S., Larsson, L., Wu, C., Furth, D., Qian, X. et al. (2019). A spatiotemporal organwide gene expression and cell atlas of the developing human heart. Cell 179, 1647-1660.

Beccari, L., Moris, N., Girgin, M., Turner, D.A., Baillie-Johnson, P., Cossy, A-C. et al. 2018. Multi-axial self-organization properties of mouse embryonic stem cells into gastruloids. Nature 562, 272-276.

Campos, I.D., Pinto, V., Souda, N., Pereira, V.H. 2018. A brain within the heart: a review on the intracardiac nervous system. J. Molec. Cell. Cardiol. 119, 1-9.

Chang, E.A., Tomov, M.L., Suhr, S.T., Luo, J., Olmsted, Z.T., Paluh, J.L., Cibelli, J. 2016. Derivation of ethnically diverse human induced pluripotent stem cell lines. Sci. Rep. 5, 15234.

Clevers, H. 2016. Modeling development and disease with organoids. Cell 165, 1586-1597.

Cortes, C., Francou, A., De Bono, C., Kelly, R.G. 2018. Epithelial properties of the second heart field. Circulation Res. 122, 142-154.

Cui, Y., Zheng, Y., Liu, X., Yan, L., Fan, X., Yong, J. et al. Single-cell transcriptome analysis maps the developmental track of the human heart. Cell Rep. 26, 1934-1950.

Das, S., Gordian-Velez, W.J., Ledebur, H.C., Mourkioti, F., Rompolas, P., Chen, H. I. et al. 2020. Innervation: the missing link for biofabricated tissues and organs. NPJ Regen. Med. 5, 11.

Desgrange, A., Le Garrec, J-L., Meilhac, S.M. 2018. Left-right asymmetry in heart development and disease: forming the right loop. Development 145, dev162776.

Drakhlis, L., Biswanath, S., Farr, C-M., Lupanow, V., Teske, J., Ritzenhoff, K. et al. 2021. Human heartforming organoids recapitulate early heart and foregut development. Nat. Biotechnol. 39, 737-746.

Fedele, L., Brand, T. 2020. The intrinsic cardiac nervous system and its role in cardiac pacemaking and conduction. J. Cardiovasc. Dev. Dis. 7, 54.

Giacomelli, E., Bellin, M., Sala, L., Meer, B.J. van, Tertoolen, L.G.J., Orlova, V.V., Mummery, C.L. 2017. Three-dimensional cardiac microtissues composed of cardiomyocytes and endothelial cells codifferentiated from human pluripotent stem cells. Development 144, 1008-1017

George, R.M., Maldonado-Velez, G., Firulli, A.B. 2020. The heart of the neural crest: cardiac neural crest cells in development and regeneration. Development 147, dev188706. 
Han, L., Chaturvedi, P., Kishimoto, K., Koike, H., Nasr, T., Iwasawa, K. et al. 2020. Single cell during foregut organogenesis. Nat. Commun. 11, 4158.

Harvey, R.P. 2002. Patterning the vertebrate heart. Nat. Rev. Genet. 3, 544-556.

Hasan, W. 2013. Autonomic cardiac innervation: development and adult plasticity. Organogenesis 9, 176193.

Hofbauer, P., Jahnel, S.M., Papai, N., Giesshammer, M., Deyett, A., Schmidt, C. et al. Cardioids reveal self-organizing principles of human cardiogenesis. Cell 184, 3299-3317.

Hookway, T.A., Matthys, O.B., Mendoza-Camacho, F.N., Rains, S., Sepulveda, J.E., Joy, D.A., McDevitt, T.C. 2019. Phenotypic Variation Between Stromal Cells Differentially Impacts Engineered Cardiac Tissue Function. Tissue Eng. Part A 25, 773-785.

Kidokoro, H., Yonei-Tamura, S., Tamura, K., Schoenwolf, G.C., Saijoh, Y. 2018. The heart tube forms and elongates through dynamic cell rearrangement coordinated with foregut extension. Development 145 , $\operatorname{dev} 152488$.

Kim, K.H., Nakaoka, Y., Augustin, H.G., Koh, G.Y. 2018. Myocardial angiopoietin-1 controls atrial chamber morphogenesis by spatiotemporal degradation of cardiac jelly. Cell Reports 23, 2455-2466.

Krishnan, A., Samtani, R., Dhanantwari, P., Lee, E., Yamada, S., Shiota, K. et al. 2014. A detailed comparison of mouse and human cardiac development. Pediatr. Res. 76, 500-507.

Lee, J., Sutani, A., Kaneko, R., Takeuchi, J., Sasano, T., Kohda, T. et al. 2020. In vitro generation of functional murine heart organoids via FGF4 and extracellular matrix. Nat. Commun. 11, 4283.

Lind, J.U., Busbee, T.A., Valentine, A.D., Pasqualini, F.S., Yuan, H., Yadid, M. et al. 2017. Instrumented cardiac microphysiological devices via multimaterial three-dimensional printing. Nat. Mater. 16, 303-308.

Ma, Z., Wang, J., Loskill, P., Huebsch, N., Koo, S., Svedlund, F.L. et al. 2015. Self-organizing human cardiac microchambers mediated by geometric confinement. Nat. Commun. 6, 7413.

Macqueen, L.A., Sheehy, S.P., Chantre, C.O., Zimmerman, J.F., Pasqualini, F.S., Liu, X. et al. 2018. A tissue- engineered scale model of the heart ventricle. Nat. Biomed. Eng. 2, 930-941.

Moon, K.R., van Dijk, D., Wang, Z., Gigante, S., Burkhardt, D.B., Chen, W.S. et al. Visualizing structure and transitions in high-dimensional biological data. Nat. Biotechnol. 37, 1482-1492.

Moris, N., Anlas, K., van den Brink, S.C., Alemany, A., Schroder, J., Ghimire, S. et al. 2020. An in vitro model of early anteroposterior organization during human development. Nature 582, 410-415.

Miao, L., Li, J., Lu, Y., Shieh, D., Mazurkiewicz, J.E., Barroso, M. et al. 2019. Cardiomyocyte orientation modulated by the Numb family proteins-N-cadherin axis is essential for ventricular wall morphogenesis. Proc. Natl. Acadm. U S A 116, 15560-15569.

Nascone, N. Mercola, M. 1995. An inductive role for the endoderm in Xenopus cardiogenesis. Development $121,515-523$. 

generation of cardiospheres promotes robust enrichment of cardiomyocytes derived from human pluripotent stem cells. Stem Cell Rep. 3, 260-8.

Olmsted, Z.T., Paluh, J.L. 2021a. Co-development of central and peripheral neurons with trunk mesendoderm in human elongating multi-lineage organized gastruloids. Nat. Commun. 12, 3020. doi: 10.1038/s41467-021-23294-7.

Olmsted, Z.T., Paluh, J.L. 2021b. Generation of human elongating multi-lineage organized (EMLO) gastruloids" Protoc. Exch. DOI: 10.21203/rs.3.pex-1441/v1.

Olmsted, Z.T., Paluh, J.L. 2021c. Stem cell neurodevelopmental solutions for restorative treatments of the human trunk and spine. Front. Cell. Neurosci. 15, 667590.

Polonchuk, L., Chabria, M., Badi, L., Hoflack, J-C., Figtree, G., Davies, M.J., Gentile, C. 2017. Cardiac spheroids as promising in vitro models to study the human heart microenvironment. Sci. Rep. 7, 7005.

Rajendran, P.S., Challis, R.C., Fowlkes, C.C., Hanna, P., Tompkins, J.D., Jordan, M.C.C. et al. 2019. Identification of peripheral neural circuits that regulate heart rate using optogenetic and viral vector strategies. Nat. Commun. 10, 1944.

Rossi, G., Broguiere, N., Miyamoto, M., Boni, A., Guiet, R., Girgin, M. et al. 2021. Capturing cardiogenesis in gastruloids. Cell Stem Cell 28, 230-240.

Sahu, S., Sharan, S. K. 2020. Translating embryogenesis to generate organoids: novel approaches to personalized medicine. iScience 23, 101485.

Sargent, P.B., Garrett, E.N. 1995. The characterization of alpha-bungarotoxin receptors on the surface of parasympathetic neurons in the frog heart. Brain Res. 680, 99-107.

Schultheiss, T.M., Xydas, S., Lassar, A.B. 1995. Induction of avian cardiac myogenesis by anterior endoderm. Development 121, 4203-4214.

Silva, A.C., Matthys, O.B., Joy, D.A., Kauss, M.A., Natarajan, V., Lai, D. et al. 2021. Co-emergence of cardiac and gut tissues promotes cardiomyocyte maturation within human iPSC-derived organoids. Cell Stem Cell 28, 2137-2152.

Steinbeck, J.A., Jaiswal, M.K., Calder, E.L., Kishinevsky, S., Weishaupt, A., Toyka, K.V. et al. 2016. Functional connectivity under optogenetic control allows modeling of human neuromuscular disease. Cell Stem Cell 18, 134-143.

Tom, V.J., Steinmetz, M.P., Miller, J.H., Doller, C.M., Silver, J. 2004. Studies on the development and behavior of the dystrophic growth cone, the hallmark of regeneration failure, in an in vitro model of the glial scar and after spinal cord injury. J. Neurosci. 24, 6531-6539. and shared determinants of cardiomyocyte contractility in multi-lineage competent ethnically diverse iPSCs. Sci. Rep. 6, 37637. 

Martinez Arias, A. 2014. Symmetry breaking, germ layer specification and axial organization in aggregates of mouse embryonic stem cells. Development 141, 4231-4242.

van den Brink, S.C., Alemany, A., van Batenburg, V., Moris, N., Blotenburg, M., Vivie, J. et al. 2020. Single-cell spatial transcriptomics reveal somitogenesis in gastruloids. Nature 582, 405-409.

van der Linde, D., Konings, E.E.M., Slager, M.A., Witsenburg, M., Helbing, W.A., Takkenberg, J.M., Roos-Hesselink, J.W. e2011. Birth prevalence of congenital heart disease worldwide. J. Amer. Coll. Cardiol. 58, 2241-2247.

Varner, V.D. Taber, L.A. 2012. Not just inductive: a crucial mechanical role for the endoderm during heart tube assembly. Development 139, 1680-1690.

Veenvliet, J.V., Bolondi, A., Kretzmer, H., Haut, L., Scholze-Wittler, M., Schifferl, D. et al. 2020. Mouse embryonic stem cells self-organize intro trunk-like structures with neural tube and somites. Science 370, eaba4937. 
bioRxiv preprint doi: https://doi.org/10.1101/2022.02.25.481991; this version posted February 25, 2022. The copyright holder for this preprint

(which was not certified by peer review) is the author/funder, who has granted bioRxiv a license to display the preprint in perpetuity. It is made available under aCC-BY-NC-ND 4.0 International license.

\section{Figure 1}

A

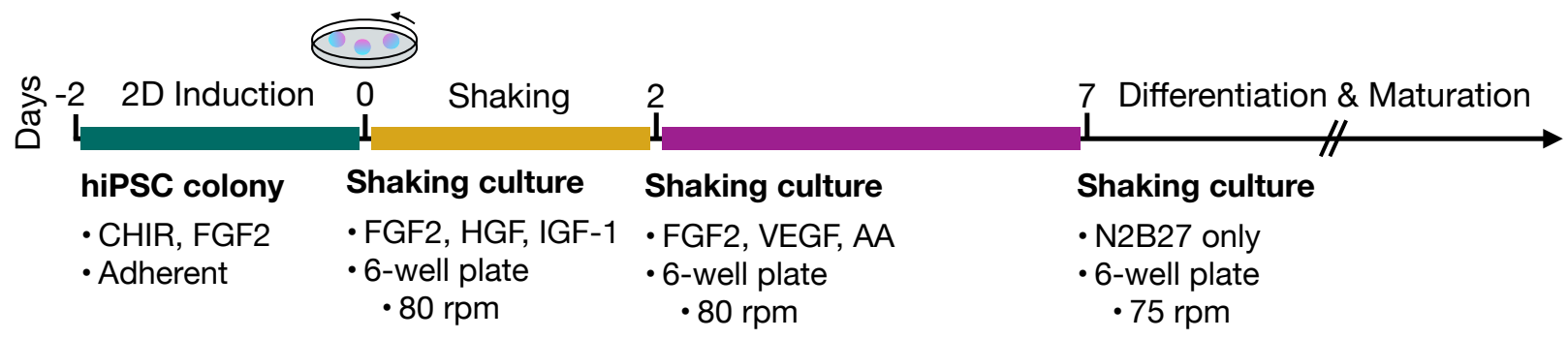

B

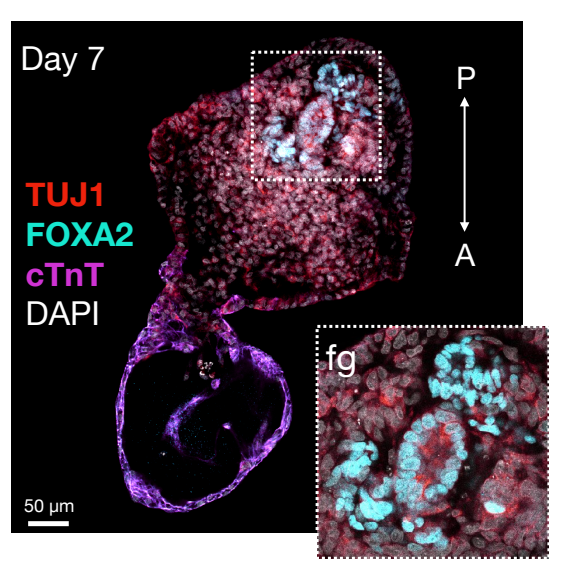

D

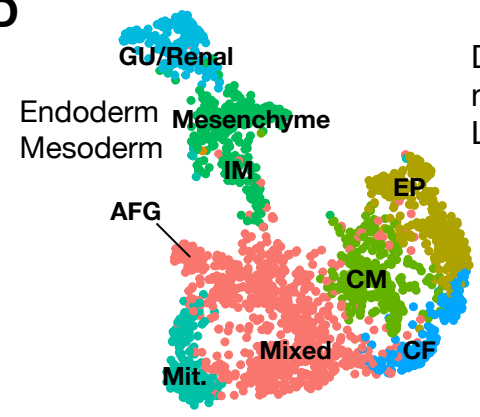

Integrated clusters:

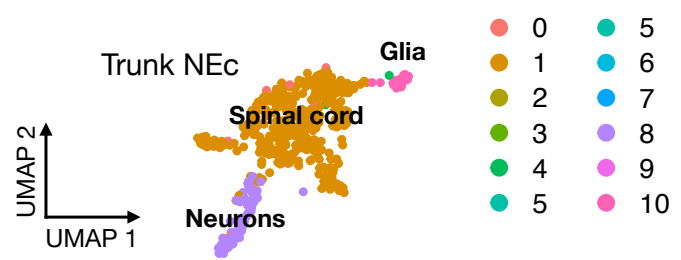

E

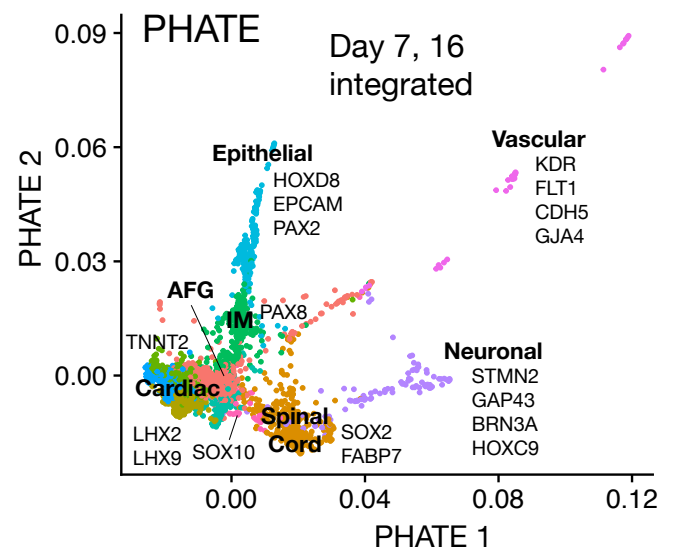

C

Day 7,16 int

$n=2,859$ cells

Line H3.1.1

VE
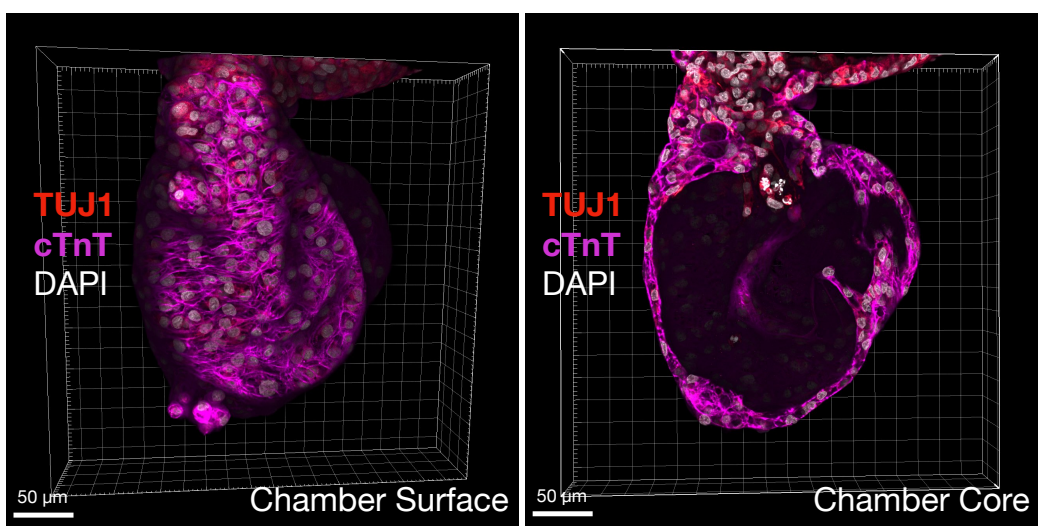

$\mathbf{F}$

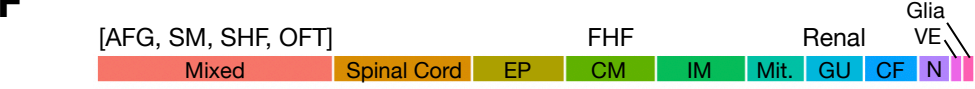

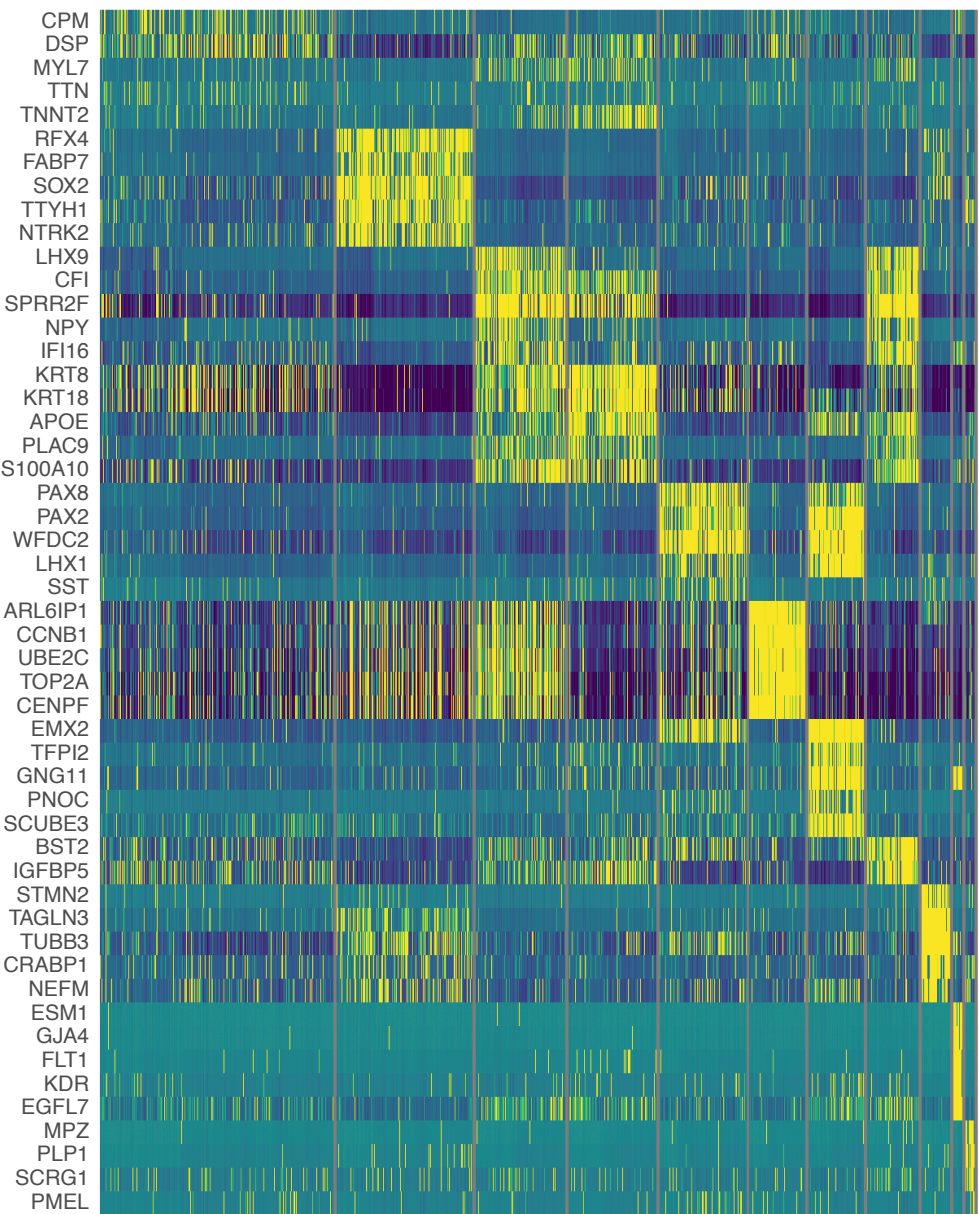


bioRxiv preprint dol: https://doi.org/10.1101/2022.02.25.481991; this version posted February 25, 2022. The copyright holder for this preprint (which was not certified by peer review) is the author/funder, who has granted bioRxiv a license to display the preprint in perpetuity. It is made available under aCC-BY-NC-ND 4.0 International license.

Figure 2

A

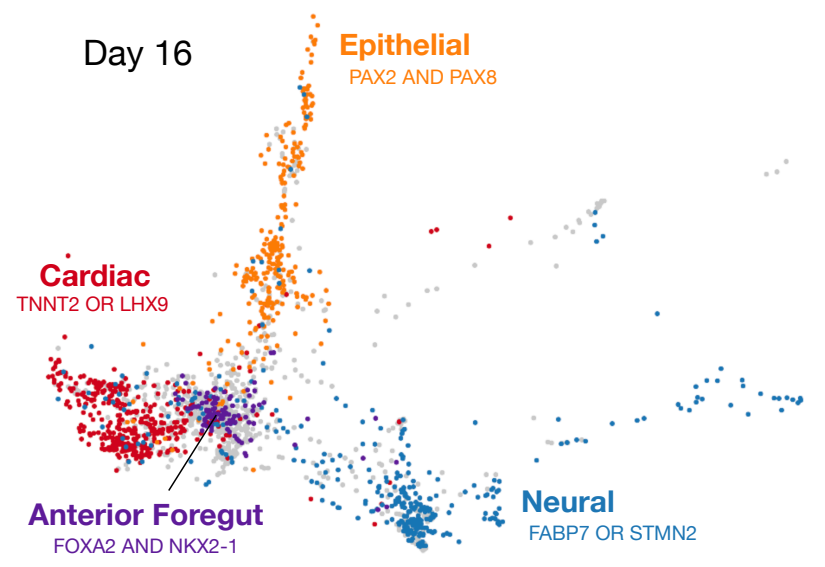

B

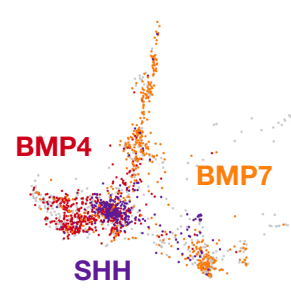

C

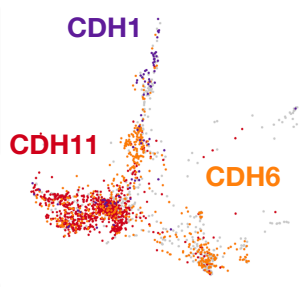

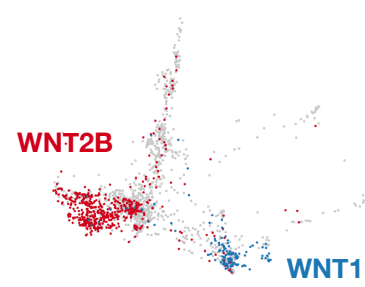

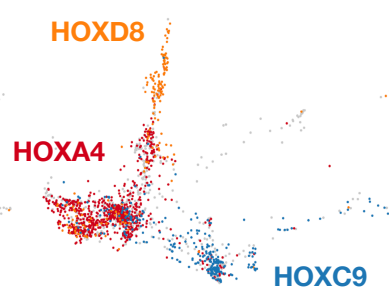

D

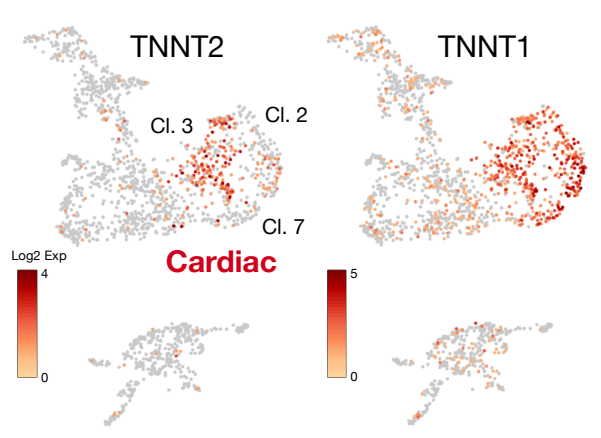

$\mathbf{E}$

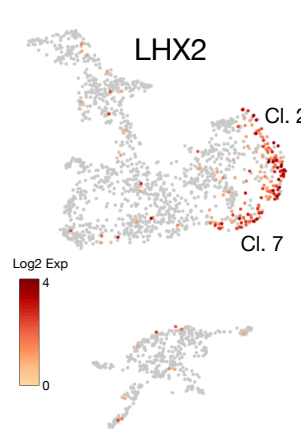

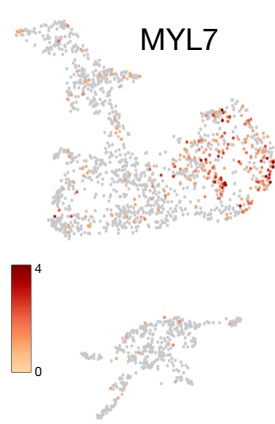
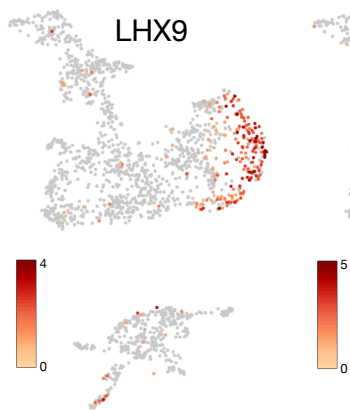
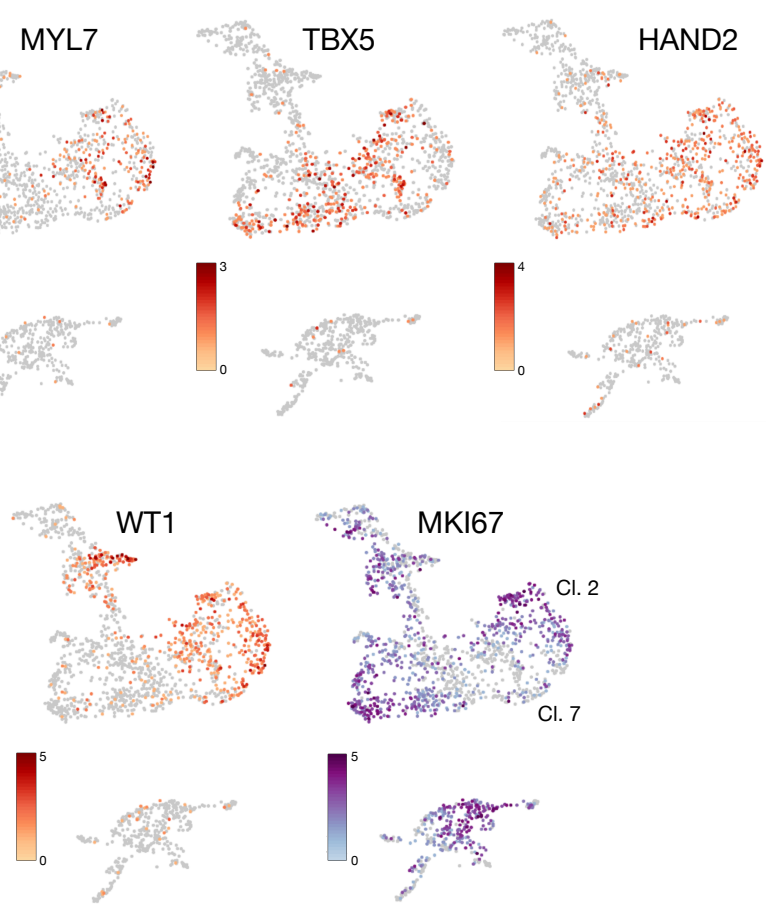
bioRxiv preprint doi: https://doi.org/10.1101/2022.02.25.481991; this version posted February 25, 2022. The copyright holder for this preprint (which was not certified by peer review) is the author/funder, who has granted bioRxiv a license to display the preprint in perpetuity. It is made available under aCC-BY-NC-ND 4.0 International license.

\section{Figure 3}

A
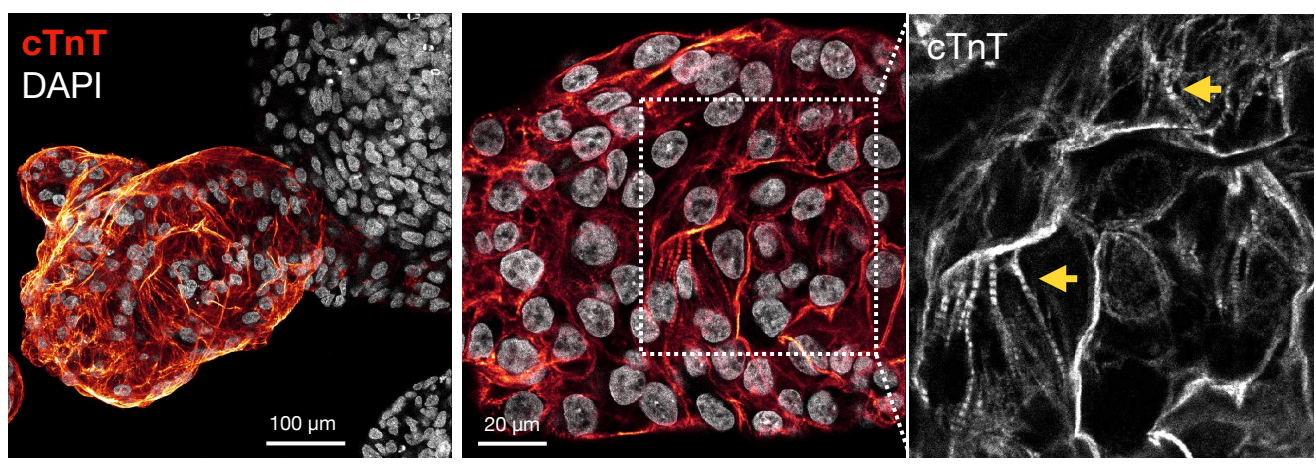

C
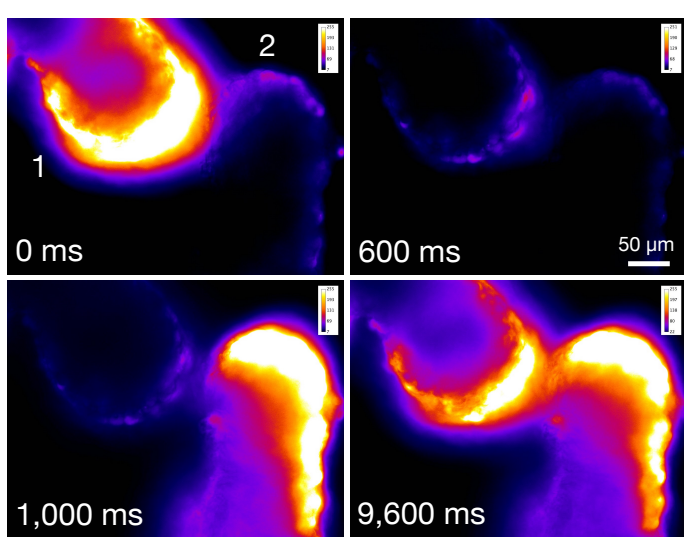

D

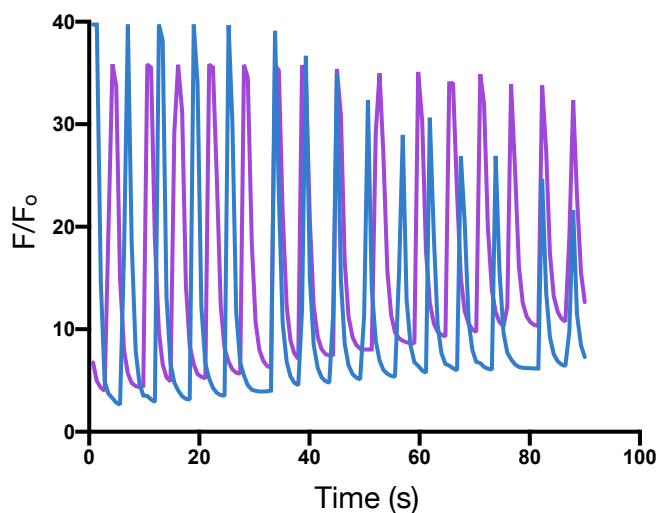

B

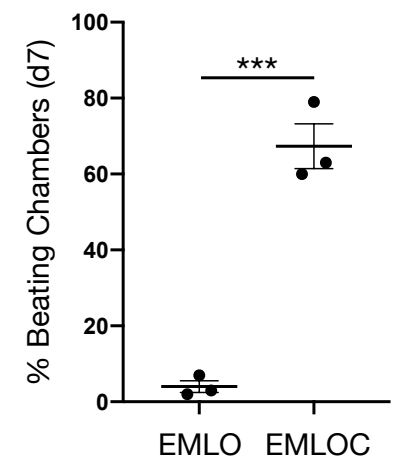

E

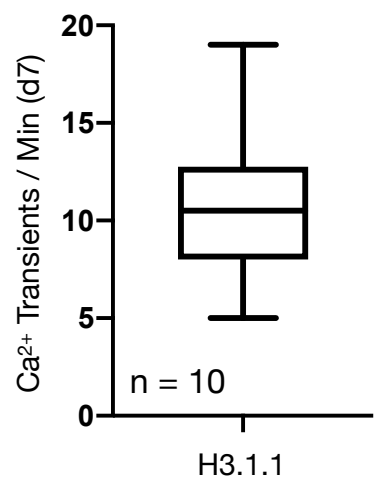

$\mathbf{F}$

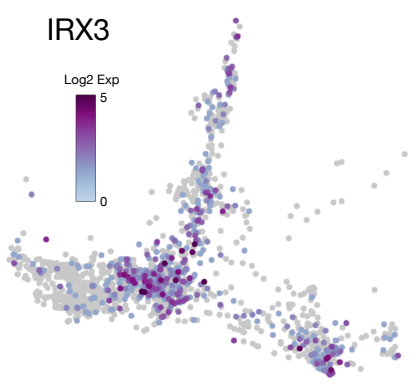

Rapid ventricular conduction

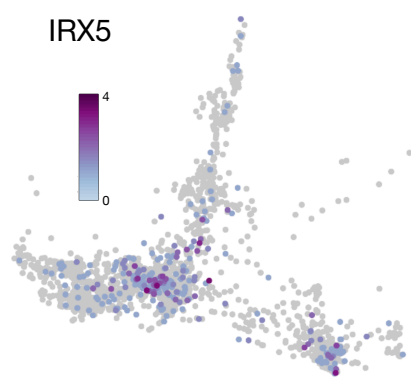

Cardiac repolarization

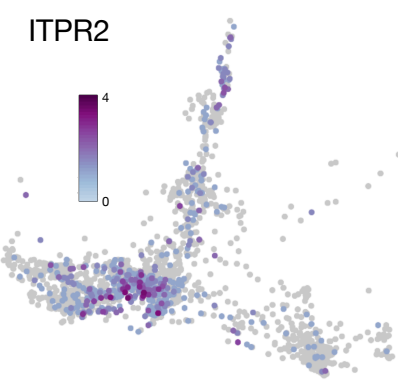

Calcium channel

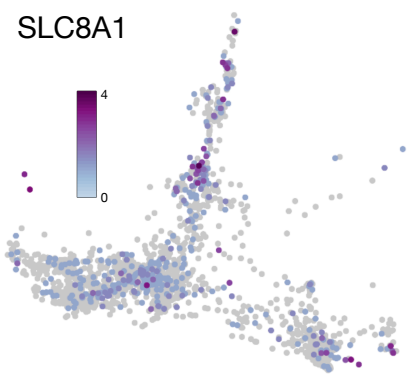

Calcium handling (NAC1) 
bioRxiv preprint doi: https://doi.org/10.1101/2022.02.25.481991; this version posted February 25, 2022. The copyright holder for this preprint

(which was not certified by peer review) is the author/funder, who has granted bioRxiv a license to display the preprint in perpetuity. It is made available under aCC-BY-NC-ND 4.0 International license.

\section{Figure 4}

A

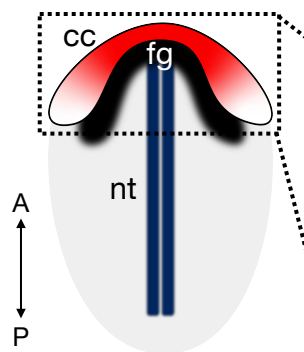

Embryo

C
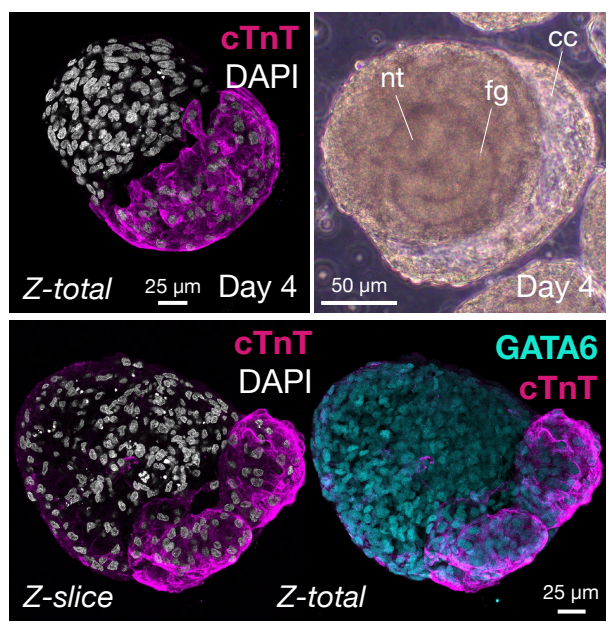

D
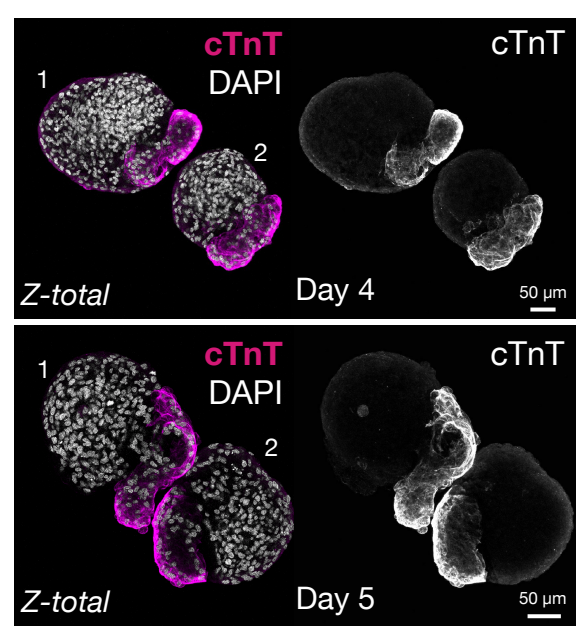

B

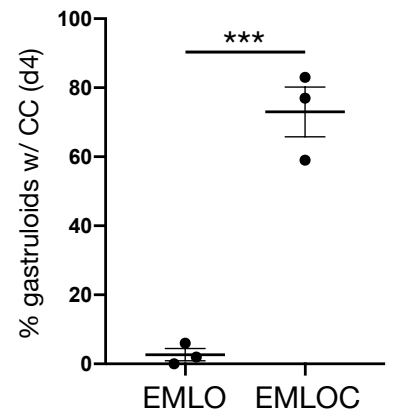

E
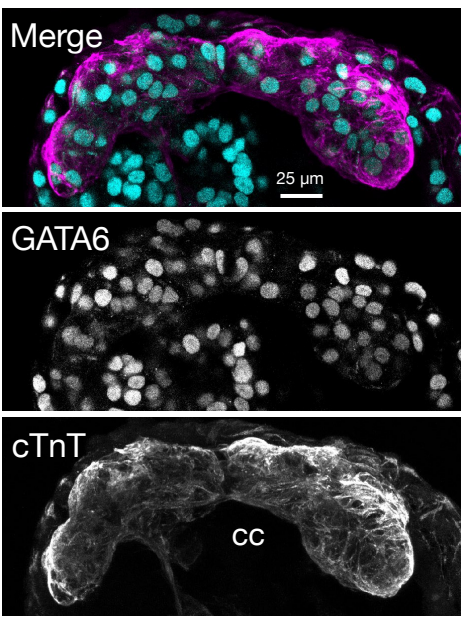

F
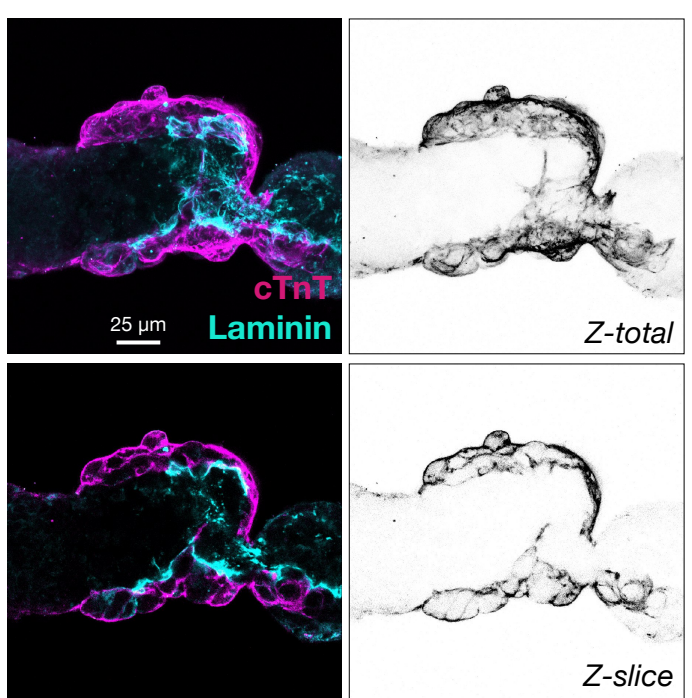

G
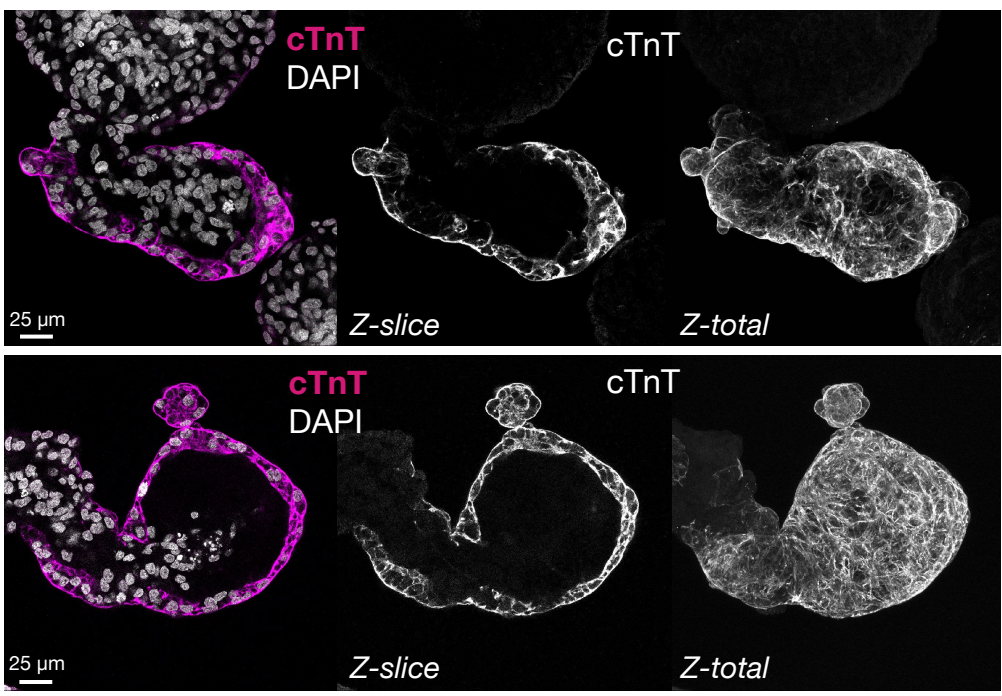
bioRxiv preprint doi: https://doi.org/10.1101/2022.02.25.481991; this version posted February 25, 2022. The copyright holder for this preprint (which was not certified by peer review) is the author/funder, who has granted bioRxiv a license to display the preprint in perpetuity. It is made available under aCC-BY-NC-ND 4.0 International license.

\section{Figure 5}

A

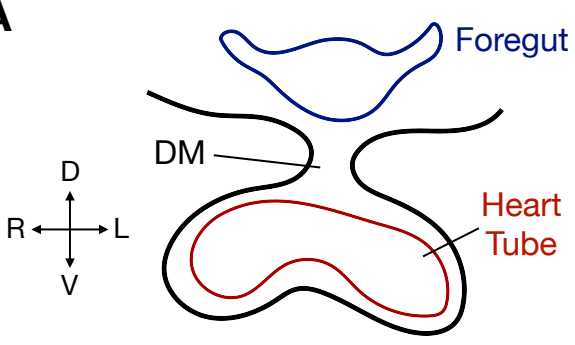

C

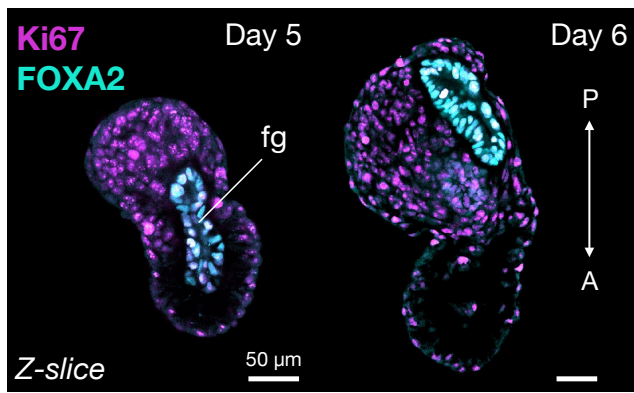

E

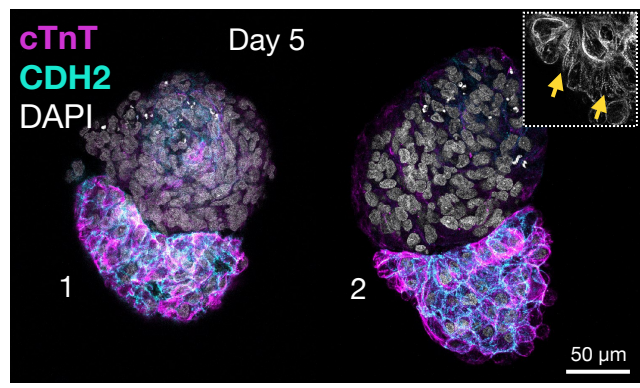

Cardiac Crescent

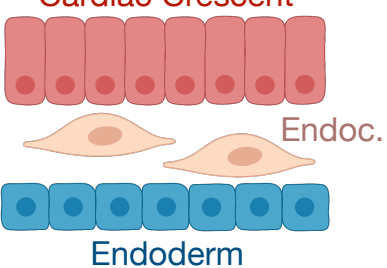

Endoderm

B

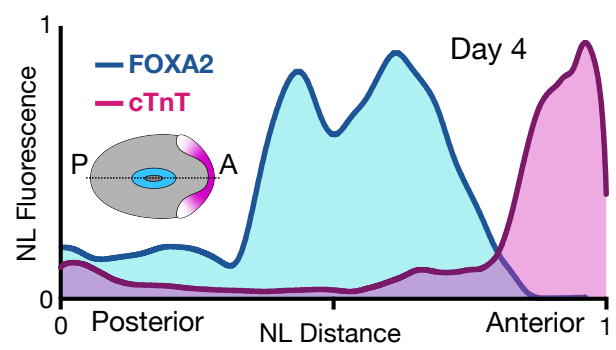

D
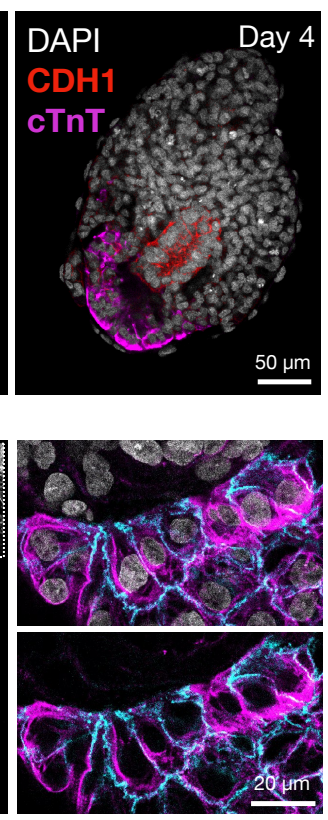
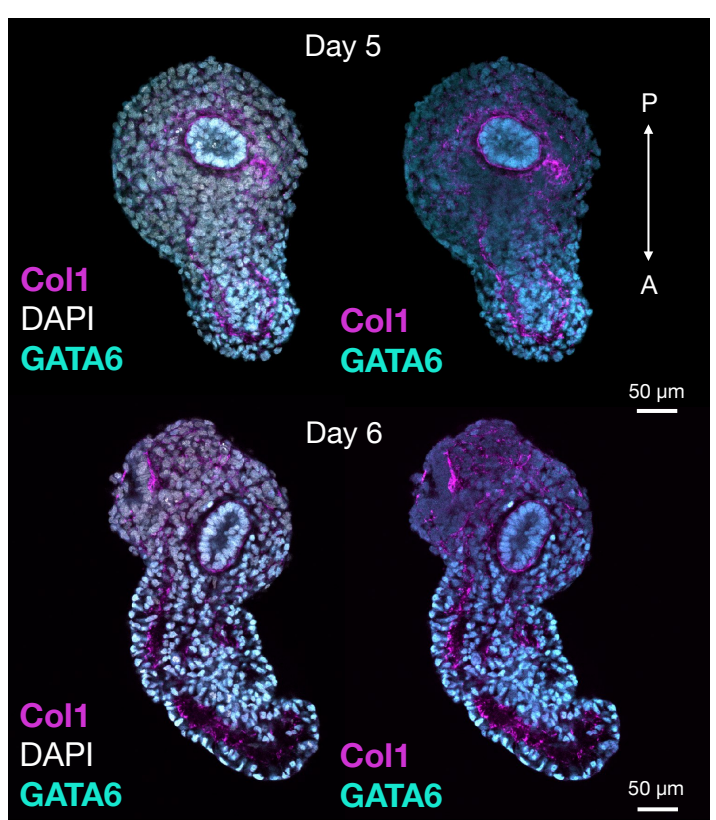
bioRxiv preprint doi: https://doi.org/10.1101/2022.02.25.481991; this version posted February 25, 2022. The copyright holder for this preprint (which was not certified by peer review) is the author/funder, who has granted bioRxiv a license to display the preprint in perpetuity. It is made available under aCC-BY-NC-ND 4.0 International license.

\section{Figure 6}

A

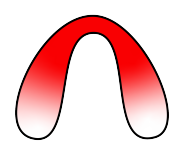

Cardiac

Crescent

C

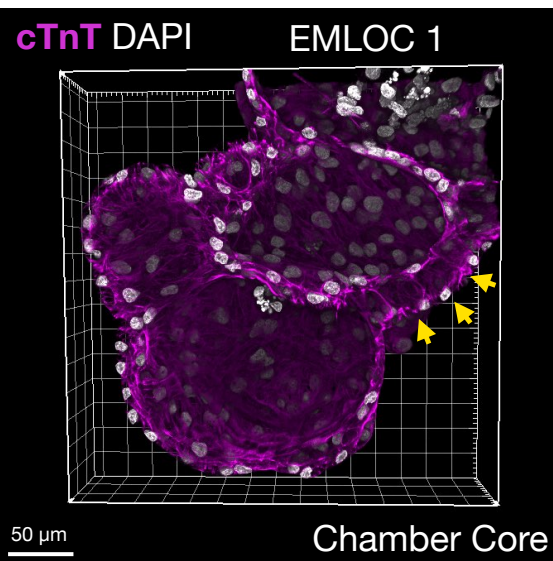

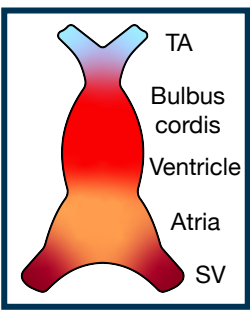

Heart Tube

Segmentation

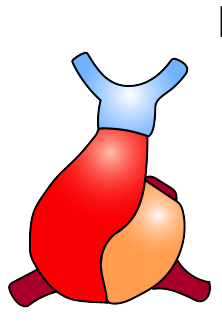

Heart Tube Looping

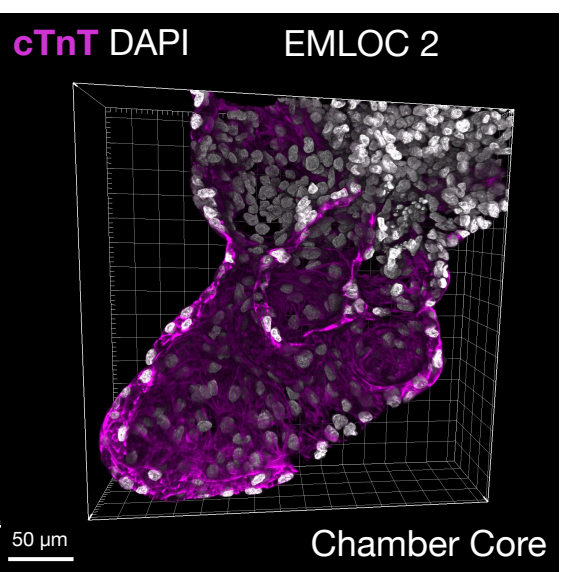

B

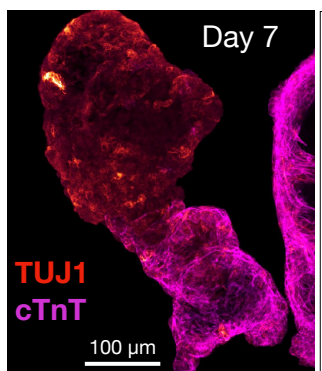

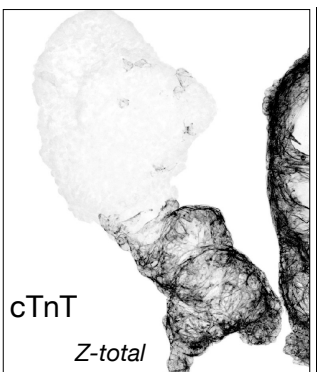

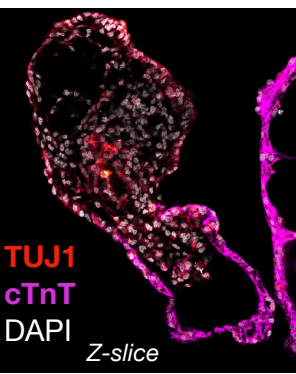

D

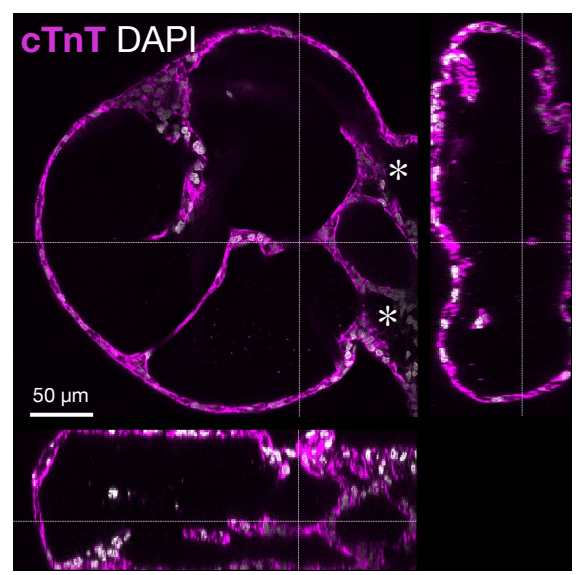


bioRxiv preprint doi: https://doi.org/10.1101/2022.02.25.481991; this version posted February 25, 2022. The copyright holder for this preprint

(which was not certified by peer review) is the author/funder, who has granted bioRxiv a license to display the preprint in perpetuity. It is made available under aCC-BY-NC-ND 4.0 International license.

\section{Figure 7}

A

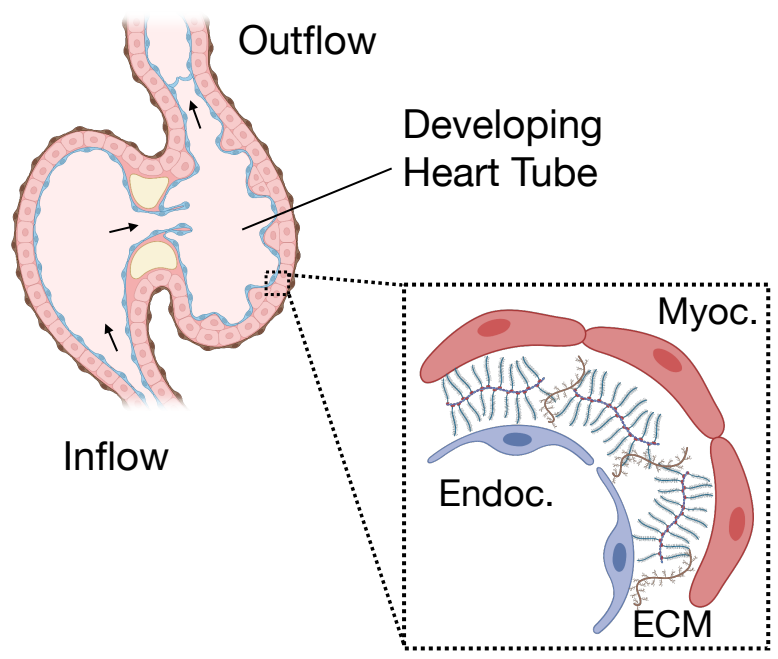

B

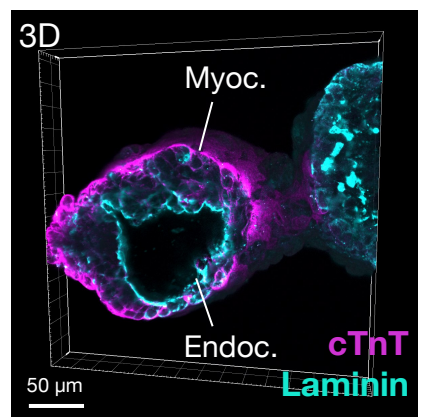

C

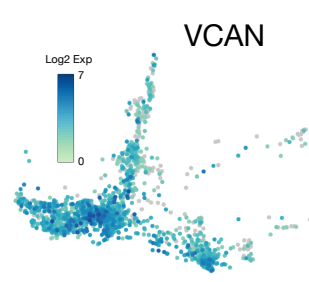

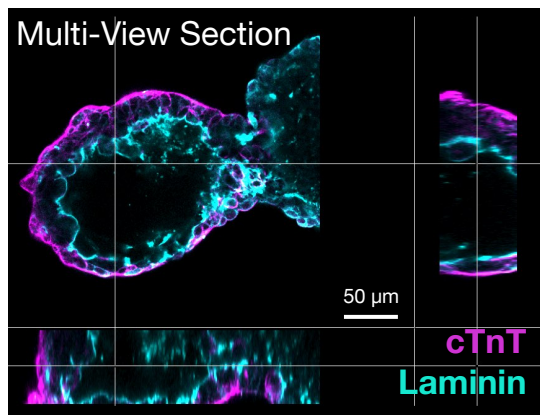
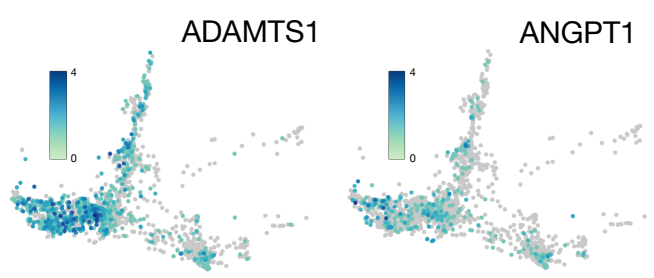

D

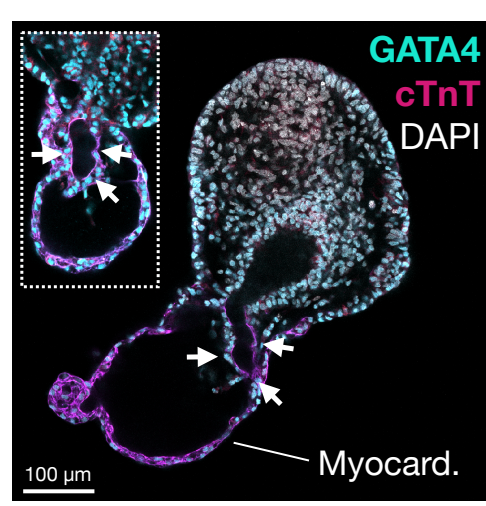

F

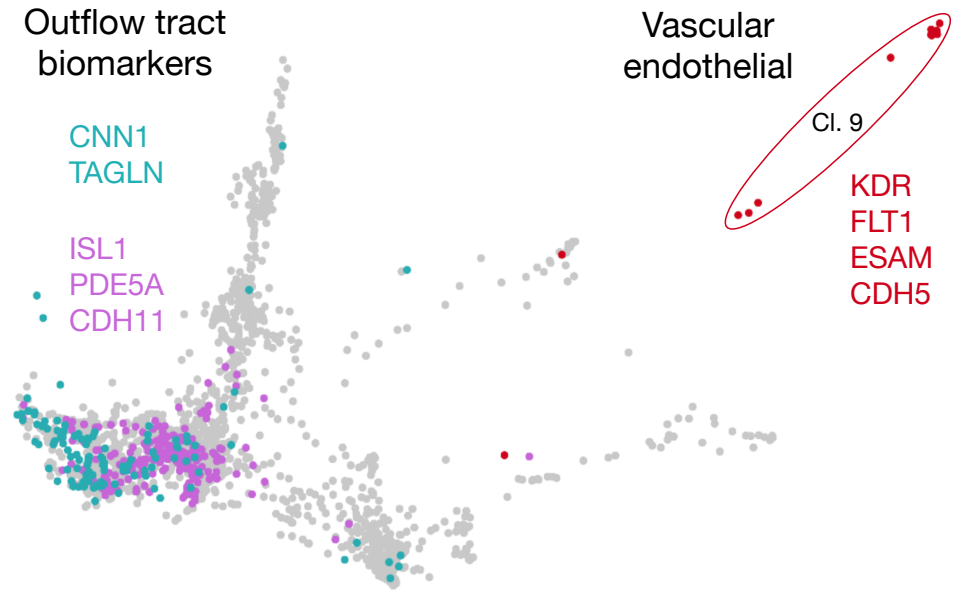

E
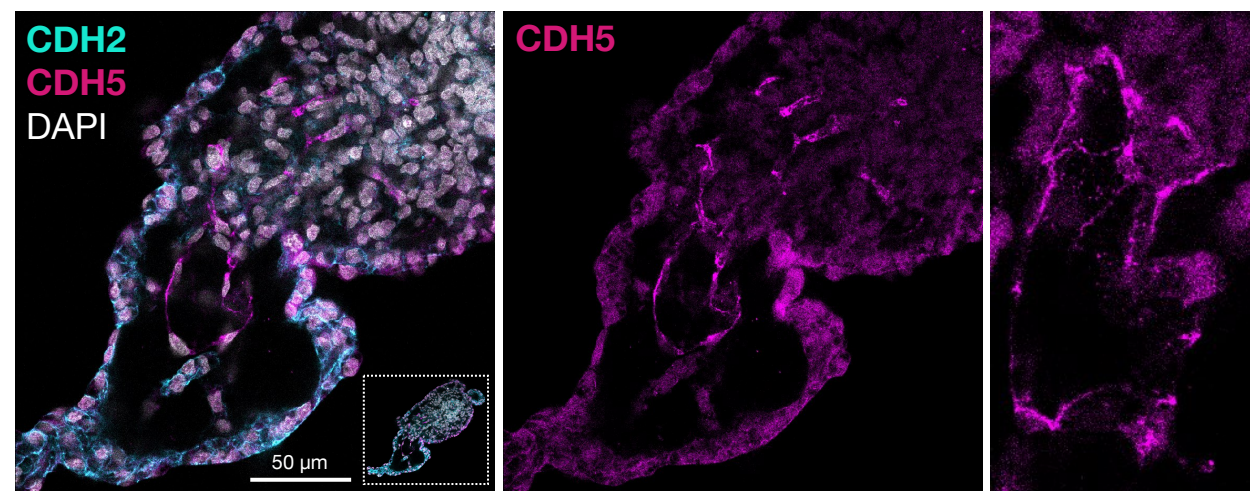

G
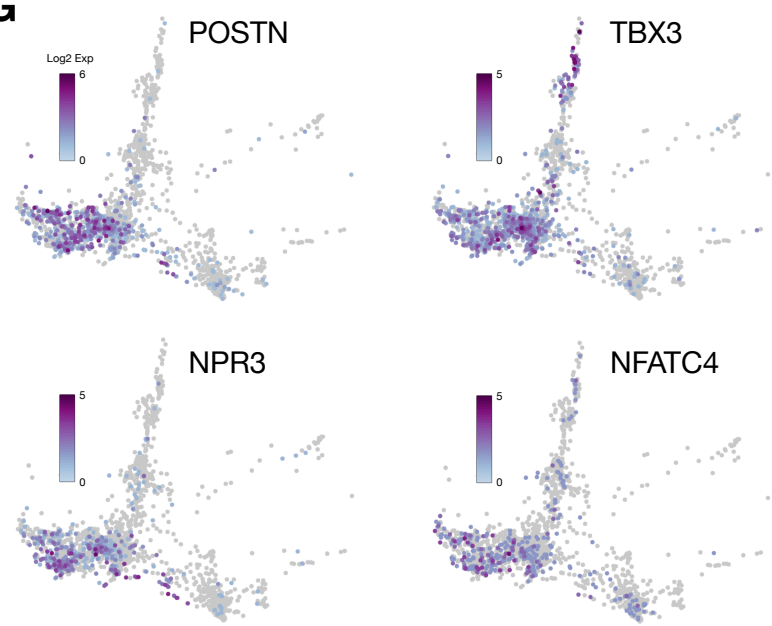

NFATC4 


\section{Figure 8}

\section{A \\ B}
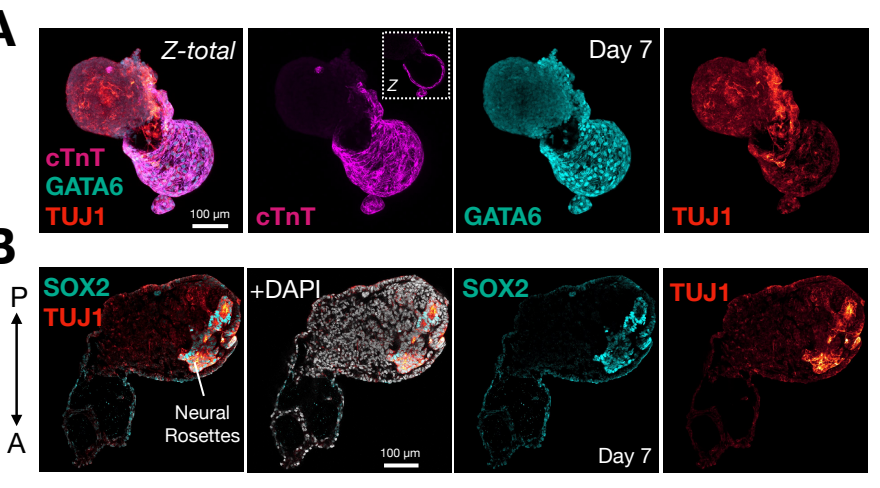

E

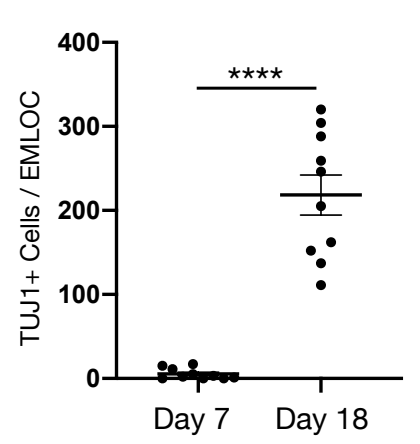

H

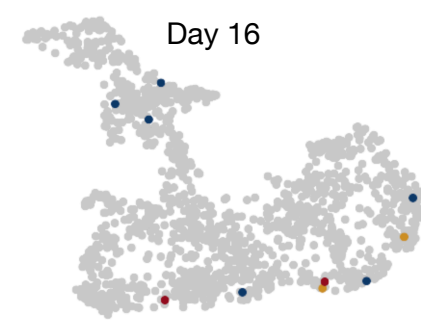

Neural progenitors ZIC1 AND RFX4

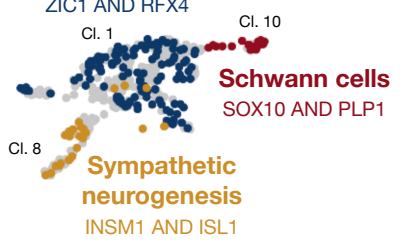

\section{J}

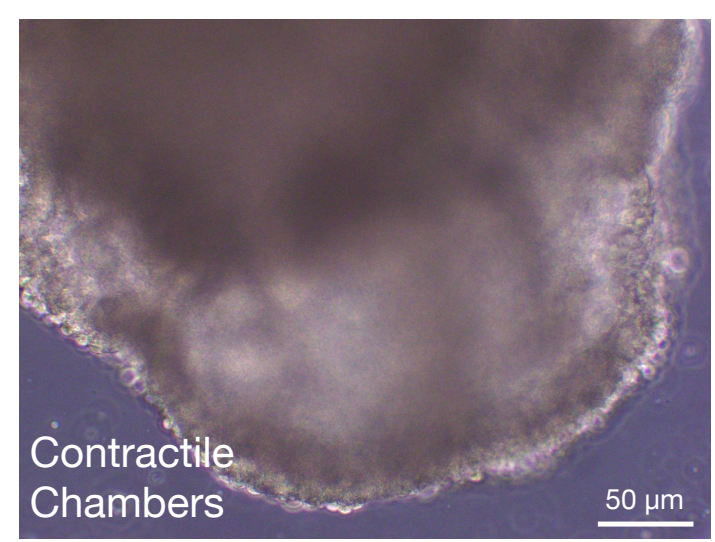

Autonomic

Sensory

Motor
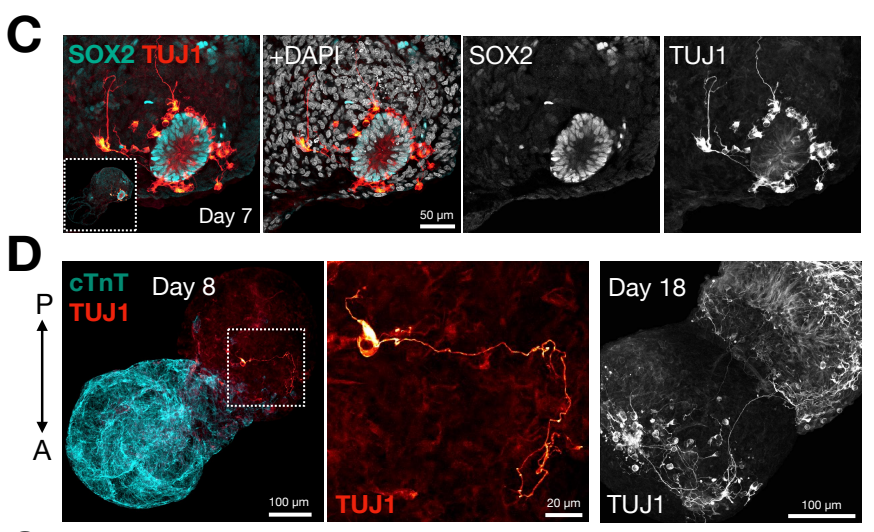

G

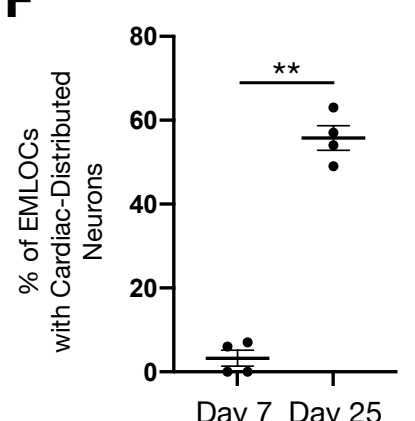

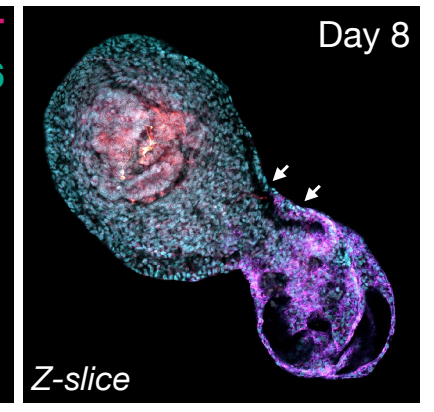
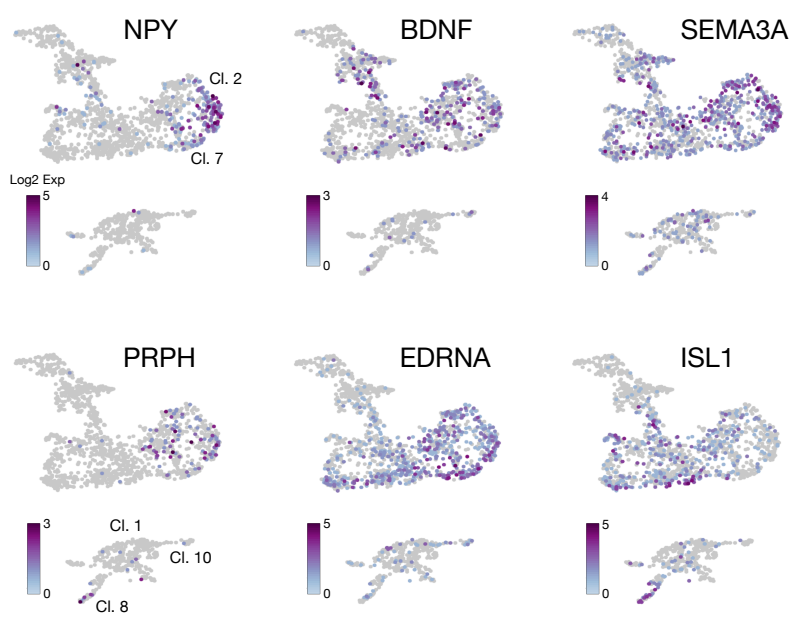

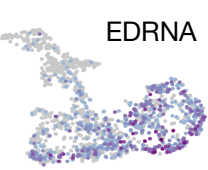

$\int_{0}^{5}-y^{2}$
ISL1

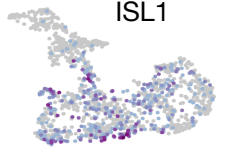

$1, y$
K

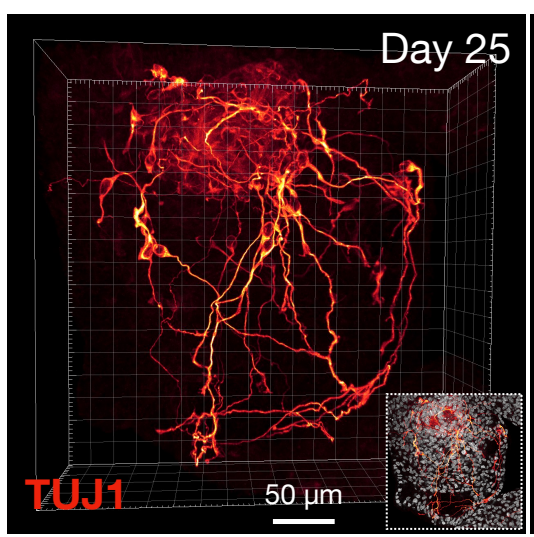

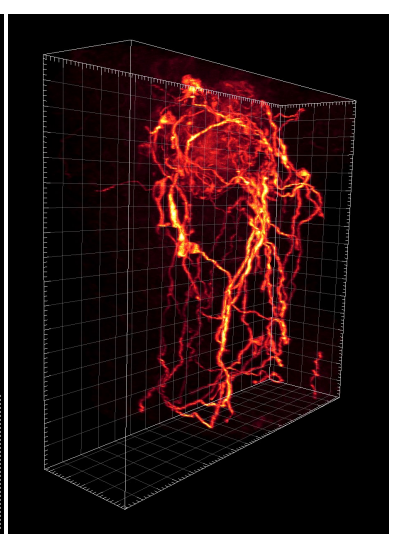

